\title{
Inversores financieros en los mercados de commodities: un modelo con dinámica de ajuste no lineal al equilibrio*
}

\author{
Diego Bastourre (BCRA, UNLP)
}

\begin{abstract}
Resumen
Los precios de los commodities han evidenciado un notable crecimiento en los últimos años, lo que ha traído consigo un sinnúmero de cambios en la economía mundial. Este trabajo busca efectuar un aporte dentro de esta temática a partir de la aplicación de un modelo autorregresivo vectorial con transición suave, enraizado en la literatura de agentes con expectativas heterogéneas. Se intenta comprender con esta metodología econométrica tanto las variables que influyen los precios de los commodities en el largo plazo, obteniendo así un precio de "equilibrio" o "fundamental", como los mecanismos de generación, amplificación y corrección de desviaciones de corto plazo respecto a tal referencia. Los resultados obtenidos sugieren una buena dosis de cautela a la hora de evaluar los precios corrientes como niveles permanentes de cara al futuro.
\end{abstract}

\begin{abstract}
Commodity prices have been growing fast in the last few years and this has caused countless economic changes worldwide. This paper looks for a place in this topic offering a smooth transition vector autoregressive model whose motivation is rooted in the heterogeneous agent based models literature. The econometric methodology aims at identifying both those variables that influence prices in the long run -obtaining in this way an "equilibrium" or "fundamental" price, and the mechanisms that start, strengthen and eventually correct short run deviations with respect to that equilibrium. The empirical results suggest that current peaks of commodities should be carefully treated. Particularly, those records levels should not been automatically taken for granted as permanent prices for the near future.
\end{abstract}

Clasificación JEL: C32, D84, Q11.

\footnotetext{
"Este trabajo constituye la tesis de la Maestría en Economía de la UNLP. Deseo agradecer a Jorge Carrera quien ha sido el director, a José Fanelli quien aceptó ser lector y a Ricardo Bebczuk quien siguió el proceso de tesis. A Javier Ibarlucia debo varias de las mejores ideas para llevar adelante el proyecto, así como numerosas sugerencias. También Fernando Toledo, Augusto Mercadier y Federico Traverso aportaron valiosos comentarios. A Omar Mendoza agradezco una fructífera instrucción sobre estimación de modelos STAR. Como es usual, los errores remanentes son mi exclusiva responsabilidad.

** e-mail: dbastourre@bcra.gov.ar. Las opiniones vertidas en este artículo son meramente a título personal y no comprometen en modo alguno la visión de las instituciones de las que el autor es miembro.
} 


\section{Introducción}

Durante las dos décadas posteriores a los grandes shocks del petróleo, la cuestión de los precios de los commodities pasó a ocupar un lugar secundario en la literatura económica teórica y empírica. Sin embargo, en los últimos cinco años, y merced a una serie de records nominales en un importante número de productos agrícolas, minerales y energéticos, la temática ha recobrado un fuerte vigor, dada la amplitud e intensidad de sus consecuencias. Ellas van desde los efectos sobre los países productores y consumidores, donde sobresale la influencia sobre la inflación, el producto, el tipo de cambio real, la política fiscal y la distribución del ingreso; hasta el impacto global que los precios tienen sobre los patrones de comercio o la organización misma del sistema monetario internacional. Frankel (2006) ha sintetizado este resurgimiento de una forma contundente: "commodity prices are back with a vengeance".

En este retorno de los commodities al centro del debate académico y de la política económica suelen ponderarse dos aspectos cuyas interconexiones no se han presentado todavía en un marco unificado.

Por un lado, se subraya que detrás del actual ciclo alcista de los precios hay un gran peso de factores fundamentales de largo plazo vinculados a la oferta y la demanda, como lo son la creciente importancia de las economías asiáticas en estos mercados, especialmente el ascenso de China en la economía internacional (FMI, 2006; Kaplinsky, 2006; Cheung y Morin, 2007); la debilidad del dólar (subrayado desde trabajos tradicionales como Ridler y Yandle, 1972; Dornbusch, 1985; o Borensztein y Reinhart, 1994); el exceso de liquidez mundial (Dooley y Garber, 2005; HSBC, 2007; Bastourre et al., 2008); la laxitud de las políticas monetarias (Frankel, 2006); o cuestiones tecnológicas como la aparición de los biocombustibles (UNCTAD, 2006; FMI, 2007; Johnson, 2007).

Por otro lado, algunos estudios recientes sostienen que ha sido la especulación financiera la causante de estas subas. Además, señalan fenómenos asociados al aumento de la profundidad con la que operan los mercados de commodities, han cambiado la morfología de las series de precios, alterando sus propiedades estócasticas (Gorton y Rouwenhorst, 2004; Erb y Harvey, 2005; Deutsche Bank, 2005; Westerhoff y Reitz, 2005; Domanski y Healt, 2007).

Dado este panorama, el objetivo del presente estudio es brindar un enfoque empírico integrador de estas esferas, lo que involucra ahondar en cuestiones reales y financieras. A tal efecto, se propone el empleo de un modelo autorregresivo vectorial con dinámica de ajuste no lineal al equilibrio. Se intenta comprender con esta metodología econométrica tanto las variables que influencian los precios en el largo plazo, obteniendo de esta manera un precio de equilibrio o "fundamental", como los mecanismos de generación y corrección de desviaciones de corto plazo respecto a tal punto de referencia.

Esta estrategia de modelación ya ha sido empleada exitosamente para testear otras hipótesis en las cuales hay múltiples interacciones entre lo real y financiero. La posibilidad de detectar múltiples regímenes a partir del grado de desalineamiento de los precios spot respecto a su valor fundamental es compatible con la presencia de agentes con expectativas heterogéneas y disposición a explotar oportunidades de arbitraje en los mercados de commodities. Desde la óptica de los policymakers, este entendimiento puede ayudar a distinguir o determinar qué efectos son permanentes o de largo plazo y cuáles movimientos se corresponden a oscilaciones transitorias, volátiles y reversibles. 
Con esta motivación, se propone la siguiente estructura para abordar el problema.

En la próxima sección se discute la relevancia de los precios de los commodities en la macroeconomía, así como la literatura que estudia sus determinantes. Se resumen también los principales hechos estilizados y hallazgos empíricos. Posteriormente, se revisan las características de los commodities vistos bajo el prisma que utilizan los inversores financieros y se puntualizan algunas tendencias recientes que hablan del incremento en la profundidad, complejidad y liquidez con la que operan estos mercados. Igualmente, se indaga allí sobre la pertinencia teórica del empleo de una técnica empírica de ajuste no lineal al equilibrio, con un modelo de agentes con expectativas heterogéneas. En la tercera parte del estudio se expone la metodología econométrica a utilizar, específicamente, la extensión vectorial de un modelo autoregresivo con transición suave $\left(\mathrm{STAR}^{1}\right)$. Seguidamente, se presenta e interpreta la propuesta empírica a través de un conjunto de tests y estimaciones y se computan las funciones de impulso-respuesta generalizadas bajo distintos regímenes para la dinámica de los precios. Se finaliza en la quinta sección con algunas reflexiones y recomendaciones de política económica.

\section{Precios de los commodities, relevancia y factores determinantes}

En todo mercado la evolución de los precios es el principal indicador de su estado de situación. Para los commodities esta no es la excepción, por lo que su importancia microeconómica o vinculada con las decisiones de producción y consumo, es evidente.

Sin embargo, se desean enfatizar en este estudio las consecuencias macroeconómicas de los movimientos de precios en estos mercados, bajo la premisa de que no estamos simplemente ante "un grupo de precios relativos más". Los efectos macroeconómicos a analizar son variados, aunque dependen del tipo de economía que se esté considerando. Como idea general, puede sostenerse que su importancia es decreciente con el nivel de ingreso por habitante, con el tamaño de la economía y con la diversificación productiva y comercial de cada país.

En las economías desarrolladas, los commodities no representan una parte significativa de la canasta de consumo de los hogares. ${ }^{2}$ En el caso de los alimentos, éste es el corolario natural de la ley de Engel (1985), hipótesis cuya estabilidad y robustez empírica se encuentra entre las más sólidamente fundadas de la ciencia económica (Houthakker, 1987; Hamilton, 2001). En cuanto a los metales, se ha planteado en la literatura una relación no lineal entre su consumo y el ingreso por habitante. La intensidad de uso se acrecienta en estadios intermedios de desarrollo para estabilizarse, o incluso decaer, una vez alcanzado un umbral de ingreso per cápita entre 15.000 y 20.000 dólares ajustados por paridad de poder adquisitivo (FMI, 2006). Incluso para el petróleo, ejemplo típico de shock de oferta en el mundo desarrollado, suele argumentarse que su importancia relativa ha descendido. La razón es que sus recientes subas no trajeron aparejados efectos tan significativos como en el pasado, tanto sobre el PIB (por la menor intensidad de uso), como sobre la inflación (por el menor pass-through). Este fenómeno

\footnotetext{
${ }^{1}$ Sigla empleada por su nombre en inglés, Smooth Transition Autoregressive Model.

${ }^{2}$ Considérese, como idea de contraste, que el peso de los alimentos en la canasta de consumo promedia un $60 \%$ en el África subsahariana, es cercano al $30 \%$ en China y representa "sólo" un $10 \%$ en los Estados Unidos (FMI, 2007).
} 
no sólo se estaría observando en los Estados Unidos, sino también en otras economías industrializadas (De Gregorio et al., 2007).

En países de desarrollo intermedio como los latinoamericanos donde la estructura productiva tiene un importante sustento en el sector primario, los commodities poseen una alta relevancia por su impacto sobre un significativo conjunto de variables. Otro caso paradigmático de la importancia de los commodities son las economías petroleras, donde la volatilidad en la cotización de este producto altera profundamente el entramado de relaciones económicas y sociales. Hay, asimismo, un grupo de países pequeños muy poco diversificados para los cuales los precios de ciertos bienes de exportación son absolutamente determinantes de la evolución macroeconómica. $^{3}$

Entre los efectos sobre variables relevantes, ha tenido amplia difusión en la literatura la relación entre términos del intercambio (TDI) y tipo de cambio real, según la cual mayores precios de exportación conducen a una apreciación real. Es por ello que la suba en los commodities se asocia muchas veces al fenómeno de "enfermedad holandesa".

Por otra parte, se ha investigado el vínculo entre producción primaria y crecimiento, sobresaliendo la idea de que la abundancia de recursos naturales puede actuar como una suerte de "maldición". En este sentido, el trabajo econométrico con regresiones de tipo crosscountry de Sachs y Wagner (1995), encuentra una asociación negativa entre la importancia relativa de los recursos naturales en la economía y la tasa de variación del producto. ${ }^{4}$ Una explicación puede provenir del "efecto voracidad" descrito por Tornell y Lane (1999). Según el mismo, cuando la estructura legal-institucional es débil los grupos de poder interactúan abusivamente por medio del proceso fiscal, amplificando más que proporcionalmente las consecuencias redistributivas de los shocks en los términos del intercambio, lo que impacta de forma adversa en el crecimiento.

También los ingresos fiscales son afectados por los acontecimientos en estos mercados, como lo demuestra la creciente importancia financiera global de los Fondos Soberanos de Riqueza (FSR), cuyo origen se asocia a la propiedad pública de commodities estratégicos. ${ }^{5}$ En otros casos, el impacto sobre las cuentas públicas ocurre por la vía impositiva, ya sea con impuestos específicos como los derechos de exportación o indirectamente vía gravámenes a la renta.

\footnotetext{
${ }^{3}$ Böwer et al. (2007) muestran que en 11 economías de África occidental y central un único commodity representa más del $87 \%$ de los ingresos totales por exportación; mientras que en otros 10 países de la región al menos la mitad de las ventas externas se explica por tan sólo dos bienes. Sin llegar a estos niveles de concentración, algunos commodities del sector agrícola tienen un rol similar en países de América Central y el Caribe.

${ }^{4}$ Contribuciones posteriores han refinado este resultado manteniendo su validez general, pero estableciendo que la asociación estadística es condicional al conjunto de variables de control que aproximen instituciones, educación, democracia o apertura. Asimismo, se ha encontrado que el signo negativo es más robusto en el caso del petróleo y los minerales en comparación con los bienes agrícolas (véase, entre otros, Sala-i-Martin y Subramanian, 2003; Stevens, 2003; o Korhonen, 2004).

${ }^{5} \mathrm{Si}$ bien no existen estimaciones precisas sobre el total de recursos administrados por los FSR, Morgan Stanley (2007) calculan su valor en alrededor de 12 billones de dólares, de los cuales no menos de dos tercios se derivarían de las cuentas de exportación de commodities; especialmente petróleo, gas natural y metales. Algunos FSR se constituyen a partir de una porción de las reservas internacionales de los Bancos Centrales que se invierten por esta vía con el objeto de incrementar rendimientos.
} 
Más controversial es la correspondencia entre los precios y el ahorro nacional. El análisis tradicional de Harbergerer (1950) y Laursen y Melzler (1950) sostiene que un shock negativo en los TDI causa una caída en el ahorro. Esta idea se mantuvo hasta comienzo de los ochenta cuando Sachs (1981) y Obstfeld (1982) la contradijeron parcialmente a partir de un enfoque intertemporal que remarcaba la importancia de analizar la duración y persistencia de los shocks para determinar la dirección del efecto final.

Otro campo explorado por la teoría es el vínculo entre precios de commodities y equidad distributiva. Sin duda, la faceta más general dentro de esta temática es la que se refiere a la distribución de beneficios derivados del comercio entre países productores de bienes primarios e industriales, en la línea inaugurada por Prebisch (1950) y Singer (1950).

Asimismo, para autores como Deaton y Larroque (1992), Deaton (1999) o Cashin y McDermott (2002) el hecho excepcional respecto a los commodities es su extrema volatilidad, lo que deja asentado un problema vinculado a la economía política de las reformas. En efecto, un ciclo de precios muy volátil plantea un desafío a la hora de implementar medidas tendientes a morigerar la dependencia de los commodities. Puntualmente, en la fase ascendente del ciclo de precios la economía experimenta un alza en las exportaciones, los ingresos fiscales y el producto, por lo que no habría incentivos políticos para correr riesgos con medidas innovadoras tendientes a la diversificación productiva con efectos visibles sólo a largo plazo; aunque tal vez se disponga de recursos con los cuales intentar abordar las mismas. En la fase baja, por otra parte, el deseo del poder político por acotar la influencia de los commodities será mayor, pero es más probable que se carezcan de los medios y del contexto favorable para llevar a cabo las acciones pertinentes.

Las líneas de trabajo expuestas hasta aquí demuestran que hay múltiples consecuencias de las oscilaciones de precios en los mercados de commodities, que varían en número e intensidad para cada economía y en cada momento del tiempo.

Otra área de estudio menos explorada se ha enfocado en las causas de los movimientos en los commodities, más que en sus efectos. Esta literatura ha propuesto un conjunto de variables macroeconómicas que, se espera, actúen como determinantes de los precios. Este tópico es el que se describe con más detalle en el siguiente apartado.

\subsection{Los determinantes de los precios de los commodities}

Dentro del conjunto de potenciales fundamentales, la relación que ha recibido más atención es la que ocurre entre el precio de los commodities y el tipo de cambio real (TCR) de Estados Unidos, conocida como "efecto denominación".

La idea subyacente, adelantada por Ridler y Yandle (1972), es que los cambios en el valor real del dólar implican fluctuaciones del poder de compra relativo internacional. Así, una apreciación del TCR de los Estados Unidos significa más poder de compra en términos de commodities en ese país y menos en el resto del mundo. Dornbusch (1985) ilustra el efecto con un modelo de oferta y demanda de commodities con dos países compradores. La expresión allí obtenida para la elasticidad del precio de los commodities al TCR estadounidense es:

$$
\frac{\partial \ln \left(\frac{P}{I P}\right)}{\partial \ln \left(\frac{I P}{e I P^{*}}\right)}=-\frac{\beta^{*}}{\left(\frac{\beta \eta}{\eta^{*}}+\beta^{*}\right)}
$$


Siendo $P$ el precio de un commodity representativo; $I P$ y $I P^{*}$ los índices generales de precios en los EE.UU. y el resto del mundo, respectivamente; $e$ es el tipo de cambio nominal multilateral (de manera que $\frac{I P}{e I P^{*}}$ es el TCR multilateral); $\eta$ y $\eta^{*}$ son las elasticidades precio de la demanda de consumo del commodity doméstica e internacional; y $\beta$ y $\beta^{*}$ representan las participaciones respectivas en la demanda total. El valor teórico de la elasticidad estaría así acotado al intervalo $[-1,0]$, convergiendo al límite superior cuanto menor es la importancia del resto del mundo como comprador.

En los estudios empíricos sobre el efecto denominación es usual encontrar una elasticidad negativa auque superior a la unidad en valor absoluto. Se interpreta entonces tal coeficiente como una sobrereacción (overshooting) de los precios de los commodities al valor real del dólar (Dornbusch, 1985; Gilbert, 1989; De Gregorio et al., 2005). ${ }^{6}$

El estado de la oferta y la demanda de commodities es otro potencial determinante de la evolución de los precios, aunque su identificación empírica también ha sido problemática.

Idealmente, en un contexto de equilibrio general, deberían considerarse simultáneamente precios y cantidades. La dificultad para superar el problema de simultaneidad, sin embargo, ha conducido a los estudios empíricos ha adoptar alguna estrategia simplificadora. Mayoritariamente se ha optado por estimar modelos que sólo incluyan la demanda de commodities, aproximada con la producción industrial de los países desarrollados. Adicionalmente, algunos autores han ampliado el modelo con proxies de oferta. Boreinsten y Reinhart (1994), por ejemplo, asumen una oferta exógena en su esquema teórico, pero incorporan dos variables proxies en su trabajo econométrico: la producción industrial de los países de la ex Unión Soviética y una variable dummy para la crisis de la década del ochenta. También Gilbert (1989) incluye los servicios de la deuda como una variable de desplazamiento de oferta. ${ }^{7}$

Dentro de los posibles factores explicativos en los precios, algunos estudios han hecho hincapié en la influencia de las condiciones monetarias. Para Frankel (2006), el hecho de observar altos precios relativos de los commodities es una clara señal de que la política monetaria global está resultando demasiado laxa. Para apoyar su argumento, presenta un modelo que incorpora el concepto de overshooting, en forma análoga a la que Dornbusch (1976) utiliza para caracterizar al tipo de cambio. En el modelo de Frankel (2006), la razón última para la sobrereacción en los commodities es la rápida velocidad de respuesta de los precios de la agricultura y la minería respecto al resto de los bienes cuando cambia la tasa de interés. Un aumento en esta variable transmitirá su influencia a través de, al menos, tres canales: i) incrementando los incentivos a la extracción presente versus la extracción futura; ii) aumentando el costo de oportunidad de las empresas por mantener inventarios; y iii) alentando a los especuladores a vender posiciones en contratos de commodities para invertir en letras del tesoro. Por los tres canales se espera entonces encontrar una asociación negativa entre tasa de interés (medida empíricamente por alguna tasa de referencia de los EE.UU.) y los precios de los commodities en términos reales.

\footnotetext{
${ }^{6}$ Las explicaciones para este valor anómalo, al menos en referencia a los modelos, apuntan generalmente a problemas de medición y/o estimación.

${ }^{7}$ La justificación es que, frente a problemas en la cuenta capital, los países afectados buscan aumentar las exportaciones de productos primarios para atenuar los problemas causados por la fuga de divisas.
} 
Una limitante de la tasa de interés como variable explicativa, es que puede no capturar apropiadamente las condiciones monetarias internacionales en un mundo donde la demanda se encuentra cada vez más dispersa. En este contexto, una alternativa tal vez más relevante es aproximar el efecto de la política monetaria mediante algún indicador de liquidez global, como en HSBC (2007) o Bastourre et al. (2008).

Un factor adicional para explicar la dinámica de precios es la productividad relativa en la producción de commodities respecto al resto de los bienes en la economía. Aquí la atención académica ha estado marcada por las controversiales ideas de Presbich (1950) y Singer (1950). Según las mismas, y en contraposición a la teoría clásica, ${ }^{8}$ la relación de intercambio entre bienes primarios e industriales tendería a deteriorarse con el paso del tiempo. Prebisch (1950) sostenía que existía una asimetría fundamental en la división internacional del trabajo por la cual los países centrales retenían todas las ganancias de sus incrementos en la productividad, mientras que las economías de la periferia transferían los frutos de su progreso tecnológico en la forma de menores precios.

La polémica en torno a esta tesis ha sido profunda y ha generado mucho trabajo empírico. La metodología principal para estudiar los precios de los commodities ha sido, hasta el presente, el análisis univariado de las series por ejemplo, mediante test de raíces unitarias o de quiebres estructurales. Al respecto, hay cierto consenso en la literatura en que los precios relativos de los bienes primarios han experimentado un deterioro en el largo plazo, si bien hay fuertes heterogeneidades para cada bien específico. Menor es el acuerdo en cuanto a la forma de este sendero descendente. Así, mientras algunos trabajos sostienen la hipótesis de una tendencia declinante a tasas aproximadamente constantes; otros autores plantean que han acaecido ciertos desplazamientos negativos o shocks estructurales en los precios, que no logran recuperarse totalmente en la fase ascendente del ciclo. ${ }^{9}$

Hasta aquí han sido descritas tanto la importancia de los precios de los commodities como algunas de las variables con potencial poder explicativo sobre su comportamiento a largo plazo. ${ }^{10}$ En la siguiente sección se estudiará cómo estos bienes se han convertido en una de las clases de activos financieros con mayor desarrollo en los últimos diez años. Luego de revisar sus características como tal tipo de activo, se integrarán las cuestiones estructurales y financieras en un marco teórico homogéneo, que incluya condiciones de ajuste al equilibrio y una dinámica de corto plazo tal que se puedan producir desvíos de esta referencia. De ser posible esta integración en un modelo empírico, podrían aislarse más claramente los movimientos transitorios en los precios de aquellos cambios de carácter más bien permanente.

\footnotetext{
${ }^{8}$ La teoría clásica heredada de Ricardo y Mill sostenía que, a causa la los rendimientos decrecientes de la tierra, el precio relativo de los productos agrícolas debía subir en el largo plazo.

${ }^{9}$ Hay numerosos trabajos que analizan el comportamiento de largo plazo de los precios relativos de los commodities. Grilli y Yang (1988) construyen varias series para el período 1990-1986, encontrando que el precio de los commodities no energéticos respecto a las manufacturas cayó un $0,6 \%$ anual en ese lapso. Véase Cuddington y Urzúa (1989); Powell (1991); Bleaney y Greenaway (1993); Lutz (1999); Cashin y McDermott (2002); o Ocampo y Parra (2003), entre otros, para una discusión más profunda en este punto.

${ }^{10}$ Vale aclarar que esta lista de determinantes no es exhaustiva y que en cada trabajo empírico el conjunto elegido se adapta a la especificidad de las hipótesis particulares.
} 


\section{Los commodities como activos financieros}

En los últimos dos años, una pregunta muy simple ha circulado entre expertos en finanzas, académicos y hacedores de política: ¿en cuánto ha contribuido la especulación financiera para explicar los altos valores, en términos históricos, de un gran número de commodities? No obstante la claridad del interrogante, su respuesta definitiva dista de resultar obvia o trivial. ${ }^{11}$

Para quienes argumentan que la especulación financiera puede estar desempeñando un rol protagónico en el último ciclo alcista de precios, hay algunas cifras muy sugestivas que parecen apoyar la creencia.

Domanski y Healt (2007), por ejemplo, señalan que el número de contratos en los mercados de derivados de commodities se triplicó entre 2002 y 2005. ${ }^{12}$ Asimismo, según datos del Bank of International Settlements, hacia mediados de 2006 el monto total de contratos en derivados alcanzaba los 6,4 billones de dólares, un valor catorce veces superior al del año 1998. Además, aun cuando el volumen operado en los mercados de futuros es pequeño en comparación con los mercados accionarios, de bonos o de divisas; resulta, en muchos casos, varias veces superior al volumen físico efectivamente producido de cada bien particular. ${ }^{13}$

En el año 2007, por su parte, esta tendencia se vio reforzada, ya que el número de contratos agrícolas subió $32 \%$ frente al año previo, mientras que para los metales y los productos energéticos las alzas fueron de $29,7 \%$ y $28,6 \%$, respectivamente. ${ }^{14}$

La intuición sugiere que la actividad especulativa puede ser un factor amplificador de los cambios fundamentales que sufren la oferta y la demanda de commodities. Así, una burbuja similar a la de los mercados accionarios o inmobiliarios es una posibilidad teórica cierta. Hasta el momento, sin embargo, la evidencia provista por la literatura ha tendido a ser más bien anecdótica o circunstancial y la falta de respuestas contundentes puede estar asociada a una serie de problemas metodológicos, partiendo incluso de la definición (y medición) misma del concepto de especulación (FMI, 2006).

En líneas generales, pueden distinguirse dos tipos de inversiones vinculadas a estos bienes. La de tipo tradicional que es la que se canaliza por medio de los mercados de futuros. La segunda forma, más reciente, tiene que ver con una serie de instrumentos de inversión cuya estrategia es la de replicar la evolución de algún índice de commodities, ya sea de carácter específico o compuesto.

\footnotetext{
${ }^{11}$ A comienzos de 2008, los continuos records del petróleo, los metales y los cereales; junto con una fuerte depreciación del dólar frente las principales monedas, llevaron a la prensa especializada a preguntarse abiertamente sobre la posibilidad de una burbuja en los precios de los commodities. Estos aparecían, hasta esa fecha, como uno de los pocos activos capaces de evitar el derrumbe de los bonos, acciones y obligaciones de los principales mercados mundiales y se plantaba, asimismo, la ocurrencia de cierta sustitución entre ambos grupos de activos.

12 Lewis (2005) brinda tanto un panorama histórico, como una descripción actual de los principales mercados mundiales de futuros de commodities.

${ }^{13}$ En el año 2005 el volumen de derivados transados fue aproximadamente treinta veces superior que la producción física para el oro, el cobre y el aluminio por ejemplo, (Domanski y Healt, 2007).

${ }^{14}$ Los datos corresponden a la Futures Industry Association, publicados en la edición del Wall Steet Journal del 28 de Febrero de 2008.
} 
En cuanto a las estrategias en los mercados de futuros, el rol de los intermediarios financieros no debe ser confundido con el interés genuino de consumidores y productores de commodities por protegerse contra el riesgo de fluctuación en los precios (FMI, 2006). Este tipo de presencia en los mercados de futuros está asociada a la operatoria comercial de agentes interesados en última instancia en el activo físico subyacente.

La actividad de traders especializados en los mercados de futuros tiene como finalidad el brindar esa cobertura. Así, la estrategia tradicional de estos actores se concentra en explotar oportunidades de arbitraje, lo que involucra básicamente tomar una posición larga (corta) en los mercados de futuros de un commodity específico y cubrirla con la posición corta (larga) en el mercado spot (Kolb, 1997). Estas oportunidades se presentan cuando el precio futuro $\left(P_{f}\right)$ se aparta del precio spot $\left(P_{S}\right)$ en una cuantía superior al costo por financiar la posición (carry cost). El arbitraje brinda así la teoría intuitiva para la determinación de $P_{f}$.

En este sentido, la alternativa al contrato futuro es simplemente esperar y comprar el bien en el mercado spot en el momento deseado. Dado que el precio que estará vigente en esa fecha es incierto en el presente, estos contratos son una manera de comprometer hoy los términos de la transacción. Para establecer su valor, los participantes del mercado tomarán como referencia el precio spot que se espera prevalezca a la fecha de finalización del contrato $\left(P_{s}^{e}\right) \cdot{ }^{15} \mathrm{Si}$ se espera un valor más alto que el nivel corriente a su vencimiento, entonces se debería observar hoy que $P_{f}>P_{s}$ (Black, 1976).

Ya que las tendencias previstas para los mercados spot se toman en cuenta ${ }^{16}$ al determinar $P_{f}$, sólo aquellos movimientos corrientes no esperados son fuente de riesgo (y retornos) para quien opera en futuros. Así, el retorno para el inversor no lo proveen las variaciones esperadas de precios, sino el premio al riesgo $(R P)$ definido como la diferencia entre $P_{f}$ y $P_{s}^{e}$.

Siempre que $P_{f}$ se establezca por encima de $P_{S}^{e}$, el vendedor de futuro obtendrá un premio al riesgo positivo. Si, por el contrario, $P_{f}<P_{s}^{e}$ entonces $R P>0$ para el comprador del contrato. Al respecto, la teoría adelantada por Keynes (1930) y Hicks (1939) postula que el premio al riesgo debería, en promedio, acumularse a favor del comprador del contrato, pues otorga a la contraparte una cobertura al asegurarle el precio futuro. ${ }^{17}$

En cuanto a las propiedades de esta inversión, Gorton y Rouwenhorst (2004) muestran que una estrategia de roll-over continuo de contratos a futuro con una cobertura garantizada por una colocación equivalente en T-bills es menos riesgosa que una inversión en acciones o en bonos

${ }^{15}$ Esto es equivalente a decir que si los agentes son forward looking el precio futuro $\left(P_{f}\right)$ debe tener incorporado la esperanza del precio spot $\left(P_{s}^{e}\right)$.

${ }^{16}$ La referencia es aquí a tendencias previstas por todos los participantes del mercado y que son de conocimiento común. Obviamente, pueden existir expectativas heterogéneas o información privada que haga que determinado precio futuro no sea previsto por la opinión promedio del mercado, pero sí lo sea por algún agente en particular.

${ }^{17}$ Situación técnicamente conocida como backwardation en el mercado de futuros. 
y ha obtenido, históricamente, un retorno similar al de estos activos. Esta estrategia con futuros ha mostrado, al mismo tiempo, otras características atractivas desde la óptica de los inversores, entre las que figuran dos muy importantes: i) una correlación negativa respecto a las acciones y los bonos; y ii) retornos que se encuentran positivamente asociados con la inflación, por lo que brindan cobertura contra la misma.

Hasta aquí se ha descrito al inversor en commodities como agente "arbitrajista" y proveedor de liquidez. Sin embargo ésta no es la única caracterización posible. En efecto, el actual ciclo alcista de precios ha estado acompañado de una mayor variedad de productos y estrategias en estos mercados.

Según Holmes (2006), un área de rápido desarrollo es el manejo pasivo del portafolio, lo cual consiste básicamente en seguir la evolución de algún índice agregado de commodities como el Goldman Sachs Commodity Index o el Dow Jones/AIG Index.

En este sentido, Bienkowski (2007) señala que el advenimiento de los Exchange Traded Commodities (ETC) en 2003 implicó que la forma de invertir en estos activos sufriera un cambio sustancial. El crecimiento de los ETC en 2005-2007 fue cercano al 1.000\% y los activos totales administrados alcanzaron, en agosto de 2007, los 28.000 millones de dólares cubriendo todos los principales tipos de commodities. Otras estrategias más complejas para tomar posiciones sobre volatilidad son posibles a partir de la aparición de las Structured Commodity Notes (McNee, 2006).

Entre las consecuencias de la presencia de los inversores financieros en los mercados de commodities, existen buenas razones teóricas para argumentar que estos actores afectan la dinámica de ajuste del precio corriente al precio de equilibrio en una forma no lineal.

Esta línea argumental se sostiene en los modelos con en agentes heterogéneos $\left(\mathrm{HAM}^{18}\right)$, y ha sido empleada exitosamente para describir el comportamiento de distintos activos financieros. Su origen se remonta a las ideas pioneras de Keynes (1936) y Simon (1957).

Según Keynes (1936) resulta dificultoso formar un juicio objetivo respecto al valor fundamental de los activos. Además, los fundamentales son relevantes sólo en la medida que un número importante entre los actores con capacidad de influencia en los precios esté efectivamente convencido de su importancia. Para un agente puede ser entonces más relevante decidir sobre la base de simples reglas con referencia directa al sentimiento generalizado del mercado. Simon (1957) en tanto, enfatiza que los individuos enfrentan límites en cuanto al conocimiento de su entorno y respecto a sus capacidades de cómputo. En contextos de racionalidad limitada e incertidumbre, los agentes encuentran satisfactorio emplear reglas aproximativas que brinden resultados positivos, aunque no necesariamente alcancen los máximos.

Hacia fines de los ochenta y principio de los noventa, estas ideas comenzaron a articularse en el marco conceptual de los HAM. Para alcanzar este avance, tuvieron un rol importante distintas encuestas de expectativas sobre paridades cambiarias, efectuadas entre operadores y especialistas financieros.

Tomando como agenda de investigación los posibles efectos amplificadores de las expectativas heterogéneas en el mercado cambiario, Frankel y Froot (1987a,b y 1990); Ito (1990); y Taylor y Allen (1992), entre otros, han mostrado que sus participantes emplean distintas estrategias de

\footnotetext{
${ }^{18}$ Por su nombre en inglés, Heterogeneous Agent Based Models.
} 
trading y modelos de pronóstico, dependiendo del horizonte de decisión. En el corto plazo, tienden a predominar las expectativas desestabilizadoras, por las cuales una depreciación corriente de la moneda, genera la creencia de mayores depreciaciones futuras. En horizontes mayores, las expectativas actúan de manera estabilizadora, primando la tendencia hacia la reversión del movimiento inicial.

Modelos intuitivos provienen de los enfoques de "fundamentalistas vs. chartistas" (Frankel y Froot, 1987a,b y 1990) o el de "operadores ruidosos" (DeLong et al., 1990a,b; Shleifer y Summers, 1990). Estos trabajos constituyen un marco relevante para entender el rol de las expectativas de precios como mecanismo de generación, amplificación y corrección de los desajustes. A continuación se presenta un esquema simple de agentes con expectativas heterogéneas para el mercado de commodities. ${ }^{19}$

\subsection{Un modelo de agentes con expectativas heterogéneas en los mercados de commodities}

El modelo supone que el cambio en el precio de mercado del período siguiente $\left(\Delta P_{t+1}\right)$ se determina por la interacción de tres tipos de agentes llamados, genéricamente, fundamentalistas $(F)$, chartistas $(C)$ y administradores de cartera $(A)$, según la expresión:

$$
\Delta P_{t+1}=a_{1} E\left(\Delta P_{t+1}^{C}\right)+a_{2} E\left(\Delta P_{t+1}^{F}\right)+a_{3} E\left(\Delta P_{t+1}^{A}\right)
$$

Donde $E\left(\Delta P_{t+1}^{C}\right), E\left(\Delta P_{t+1}^{F}\right)$ y $E\left(\Delta P_{t+1}^{A}\right)$ son las expectativas de cambio en los precios para cada clase de agente y $a_{1}, a_{2}, a_{3}$ son ponderadores fijos que denotan la importancia de los distintos grupos.

El análisis de los fundamentalistas se sostiene en la premisa de que los precios revertirán a su valor de equilibrio a largo plazo. La especificación para sus expectativas es la siguiente:

$$
E\left(\Delta P_{t+1}^{F}\right)=-\alpha\left(P_{t}-F_{t}\left(X_{t}\right)\right) \quad \alpha>0
$$

Definiendo a $F_{t}$ como el precio de equilibrio fundamental del commodity (o una canasta de commodities) vigente en $t$. Este es función de un vector de variables $\left(X_{t}\right)$ señaladas en la primera sección, tales como el tipo de cambio real de los EE.UU., el estado de la demanda, la liquidez internacional, etc.

La regla (3) sugiere comprar el commodity cuando el precio corriente sea inferior al valor fundamental y vender en el caso contrario. Nótese que no es necesario suponer que los fundamentalistas conocen con certeza el verdadero valor del commodity a largo plazo, pero sí debería ocurrir, al menos, que cuenten con una estimación consistente de dicho valor. Podría asumirse, por ejemplo, que estos agentes tienen un conocimiento imperfecto del verdadero modelo porque existe incertidumbre sobre el valor de los parámetros; pero construyen expectativas con una regla de aprendizaje basada en una regresión econométrica por mínimos cuadrados sin cometer errores sistemáticos (Bray y Savin, 1986 y Fourgeaud et al., 1986).

Los chartistas por su parte, emplean el análisis técnico intentando seguir las tendencias actuales en los precios. Una formalización posible de tal estrategia es:

\footnotetext{
${ }^{19}$ Se trata de una adaptación estilizada de los trabajos de Frankel y Froot (1987a,b, 1990) empleando una función de ajuste exponencial y un precio fundamental que varía en el tiempo.
} 


$$
E\left(\Delta P_{t+1}^{C}\right)=\delta\left(P_{t}-P_{t-1}\right) \quad \delta>0
$$

De esta manera, siempre que los precios se incrementen los chartistas tomarán una posición larga en commodities.

Se supone que los administradores de cartera tienen una ventaja de información, en el sentido de que conocen las características del mercado en el que están operando. Para tomar provecho de dicha circunstancia, los agentes tipo $A$ ajustan su estrategia utilizando un promedio ponderado de las expectativas dadas en (3) y (4):

$$
E\left(\Delta P_{t+1}^{A}\right)=\left(1-w_{t}\right) E\left(\Delta P_{t+1}^{C}\right)+w_{t} E\left(\Delta P_{t+1}^{F}\right) \quad 0 \leq w_{t} \leq 1
$$

Aquí es determinante el rol de la variable $w_{t}$ que, se asume, ajusta de forma endógena regulando el impacto de las expectativas de los operadores tipo $F$ y $C$ sobre la conducta del administrador de cartera en cada momento $t$. En este modelo simple $w_{t}$ proporciona la no linealidad en la ecuación del cambio en los precios (2). Puntualmente, se propone emplear la siguiente función:

$$
w_{t}=1-\exp \left(-\left(\gamma\left(P_{t-d}-F_{t-d}\left(X_{t-d}\right)\right)^{2}\right)\right) \quad \gamma>0, d \geq 0
$$

La intuición para la ecuación (6) es que el desalineamiento entre los precios y sus determinantes, con algún grado de rezago dado por $d$, es la variable que gobierna el peso que los operadores $A$ le asignan a cada modelo de formación de expectativas en su estrategia.

Nótese así que cuando $F_{t-d} \cong P_{t-d}$, ocurrirá que $w_{t}$ tomará un valor muy pequeño. Esto significa que un desalineamiento bajo alentará a los administradores de cartera a emular la conducta de los chartistas.

Un caso extremo que puede imaginarse es aquel en el cual $F_{t-d}=P_{t-d}\left(w_{t}=0\right)$ y $d=0$. Reemplazando apropiadamente estas restricciones, el cambio en los precios estará dado por:

$$
\Delta P_{t+1}=\left(a_{1}+a_{3}\right) E\left(\Delta P_{t+1}^{C}\right)=\left(a_{1}+a_{3}\right) \delta\left(\Delta P_{t}\right)
$$

Así, la regla (7) sugeriría construir un modelo econométrico de precios de los commodities estrictamente autorregresivo.

A medida que la discrepancia entre $F_{t-d}$ y $P_{t-d}$ se acentúa, la posición de los traders tipo $A$ comienza a ser cada vez más contraria a la tendencia en el precio corriente de mercado. Nuevamente, el caso límite sería aquel en el que $-\left(\gamma\left(P_{t-d}-F_{t-d}\right)^{2}\right) \rightarrow-\infty$, que implica que $w_{t}=1$ y $E\left(\Delta P_{t+1}^{A}\right)=E\left(\Delta P_{t+1}^{F}\right)$. Luego de las sustituciones pertinentes, la dinámica de precios tendrá la forma de un modelo de corrección de errores, esto es:

$$
\Delta P_{t+1}=a_{1} \delta\left(\Delta P_{t}\right)-\left(a_{2}+a_{3}\right) \alpha\left(M_{t}\right)
$$

En donde se ha definido que $M_{t}=P_{t}-F\left(X_{t}\right)$.

También es importante dentro de este esquema el rol del parámetro $\gamma$. Este regula la velocidad con la que los administradores de cartera ajustan sus ponderadores. Si $\gamma$ es muy alto, por 
ejemplo, aun un pequeño desalineamiento acercará mucho las creencias de los agentes $A$ a la de los fundamentalistas.

La forma general para la dinámica de precios puede obtenerse combinando (3), (4), (5) y (6) en (2):

$$
\begin{aligned}
& \Delta P_{t+1}=a_{1} \delta \Delta P_{t}-\left(a_{2}+a_{3}\right) \alpha M_{t}+ \\
& a_{3} \delta \exp \left(-\gamma\left(M_{t-d}\right)^{2}\right) \Delta P_{t}+a_{3} \alpha \exp \left(-\gamma\left(M_{t-d}\right)^{2}\right) M_{t}
\end{aligned}
$$

Así, la ecuación del cambio en el precio de los commodities depende de varios factores. En primer término, del propio pasado de la variable, que tiene un rol autónomo e interactúa también con el desalineamiento pasado. Por otro lado, se adiciona un término de ajuste al equilibrio de carácter estándar. Se incorpora asimismo un componente no lineal de ajuste, determinado por la historia de la brecha entre el precio corriente y el precio fundamental.

El objetivo de las próximas dos secciones es estimar una versión aumentada del modelo no lineal (9) para un índice compuesto de commodities expresado en términos reales.

Se mostrará cómo una variante particular de los modelos autorregresivos con transición suave (STAR) presenta características muy similares a las indicadas por la función (9). Se trata de un modelo aumentado pues se permitirá tanto un mayor número de rezagos del cambio en los precios, como la presencia de múltiples ecuaciones (una estructura de sistema). Con esta especificación empírica será posible estudiar el equilibrio de los fundamentales, las propiedades del ajuste hacia el mismo y los efectos de corto plazo de los shocks de las variables incluidas en el vector $X_{t}$. A continuación se describe la metodología econométrica. Los resultados empíricos se tratarán en la cuarta parte del trabajo.

\section{Metodología econométrica: modelos autorregresivos con transición suave}

En las últimas décadas se ha observado un interés creciente por el uso de técnicas econométricas no lineales. Entre ellas, los modelos con cambios de régimen tienen como atractivo la incorporación de una regla de conducta que describe el cambio entre estados. Se permite así la coexistencia de distintas propiedades para una serie de tiempo en cada uno de los regímenes. La ley que regula el cambio de estado puede, a su vez, ser determinística o estocástica. En el primer caso, el régimen se determina por los valores pasados de una (o más) variables observables y es conocido con certeza por los agentes económicos. El estado es estocástico, por su parte, cuando sólo se conoce con cierta probabilidad el régimen vigente en cada momento del tiempo.

El modelo autorregresivo con régimen determinístico más simple es el de cambios abruptos, desarrollado por Tong (1978), Tsay (1989) y Tong (1990). Sintéticamente, consiste en comparar la variable de transición $\left(T V_{t}\right)$ con un umbral o threshold $(c)$, a efectos de descomponer un modelo lineal en dos sub-modelos lineales. Suponiendo una especificación autorregresiva sin variables explicativas se tendrá:

$$
y_{t}=\begin{array}{ll}
\left(\phi_{1,0}+\phi_{1,1} y_{t-1}+\ldots+\phi_{1, p} y_{t-p}\right)+\varepsilon_{t} & \forall y_{t} \leq c \\
\left(\phi_{2,0}+\phi_{2,1} y_{t-1}+\ldots+\phi_{2, p} y_{t-p}\right)+\varepsilon_{t} & \forall y_{t}>c
\end{array}
$$


En la expresión (10), el cambio de estado está dado por el propio valor de la variable respecto al umbral y ocurre de manera abrupta. La idea de que la transición entre estados puede hacerse paulatinamente constituye un avance importante dentro de esta literatura.

Contemplando como caso particular al modelo con cambios abruptos, la hipótesis general de transición suave permite que los distintos agentes económicos reaccionen de manera no simultánea frente a una perturbación económica o ante la llegada de nueva información. También es válida esta estrategia cuando los efectos de los cambios estructurales se van materializando lentamente en la economía.

Asimismo, siempre que la teoría o la intuición sugieran que una relación entre variables puede ser válida bajo alguna circunstancia, pero que se va modificando al alterarse ésta última, un modelo con transición suave puede constituir un punto de partida apropiado. Chan y Tong (1986); Granger y Teräsvirta (1993); Teräsvirta (1994); o Franses y van Dijk (2000) son referencias fundamentales del enfoque.

La representación de un modelo autorregresivo con transición suave de orden $p, \operatorname{STAR}(p)$, es la siguiente: ${ }^{20}$

$$
\begin{aligned}
& y_{t}=\left(\phi_{1,0}+\phi_{1,1} y_{t-1}+\ldots+\phi_{1, p} y_{t-p}\right)\left(1-F\left(T V_{t-d} ; \gamma, c\right)\right)+ \\
& \left(\phi_{2,0}+\phi_{2,1} y_{t-1}+\ldots+\phi_{2, p} y_{2-p}\right) F\left(T V_{t-d} ; \gamma, c\right)+\varepsilon_{t}
\end{aligned}
$$

O, alternativamente:

$$
\begin{aligned}
& y_{t}=\left(\phi_{1,0}+\phi_{1,1} y_{t-1}+\ldots+\phi_{1, p} y_{t-p}\right)+ \\
& \left(\lambda_{2,0}+\lambda_{2,1} y_{t-1}+\ldots+\lambda_{2, p} y_{2-p}\right) F\left(T V_{t-d} ; \gamma, c\right)+\varepsilon_{t}
\end{aligned}
$$

Donde se satisface que:

$$
\begin{aligned}
& \lambda_{2,0}=\phi_{2,0}-\phi_{1,0}, \lambda_{2,1}=\phi_{2,1}-\phi_{1,1}, \ldots, \lambda_{2, p}=\phi_{2, p}-\phi_{1, p} \\
& E\left(\varepsilon_{t} \mid \Omega_{t-1}\right)=0 \\
& E\left(\varepsilon_{t}^{2} \mid \Omega_{t-1}\right)=\sigma^{2} \\
& \Omega_{t-1}=\left(y_{t-1}, \ldots, y_{t-p}\right)
\end{aligned}
$$

En las ecuaciones (11) y (12), la expresión $F\left(T V_{t-d} ; \gamma, c\right)$ se conoce con el nombre de función indicadora o de transición. Se trata de una función continua y que tiene como imagen el intervalo cerrado $[0,1] \cdot{ }^{21}$ El parámetro $\gamma$ mide cuán gradual es el ajuste entre regímenes, al tiempo que $c$ es el parámetro de transición, que traza una línea divisoria a partir de la cual comienza a materializarse el cambio de estado. Por su parte, $T V_{t-d}$ se refiere a la variable de transición con $d$ rezagos.

\footnotetext{
${ }^{20}$ Para simplificar la presentación se omiten, momentáneamente, las extensiones en las cuales se incorporan otras variables explicativas, así como los modelos multiecuacionales o sistemas.

${ }^{21}$ Ambas propiedades marcan la diferencia de los modelos regresivos con transición suave respecto a los modelos con cambios abruptos, en los cuales la función indicadora es directa y toma valores 001 .
} 
Técnicamente, hay muy pocas restricciones sobre el tipo de variable que puede actuar como $T V_{t-d}$. Algunas opciones habituales son emplear los rezagos de la propia variable endógena, una variable exógena, una función de variables endógenas y/o exógenas o una tendencia temporal (van Dijk et al., 2002).

En ciertas ocasiones, sin embargo, la hipótesis teórica que se desea estudiar sugiere qué variable puede considerarse como determinante de la transición. Tal es el caso del estudio de los precios de los commodities y su posible patrón de corrección no lineal al equilibrio.

Según lo subrayado en la sección anterior, la restricción teórica a la que deberá ajustarse el modelo empírico es que la brecha o desalineamiento entre el precio corriente y el valor fundamental (con algún rezago) no sólo actuará como determinante del cambio en los precios, sino también como variable que gobierne la transición entre estados.

En cuanto a la forma de $F\left(T V_{t-d} ; \gamma, c\right)$, lo usual es postular o bien una función logística o bien una exponencial, esto es:

$$
\begin{aligned}
& F\left(T V_{t-d} ; \gamma, c\right)=\frac{1}{1+\exp \left(-\gamma\left(T V_{t-d}-c\right)\right)}, \gamma>0 \\
& F\left(T V_{t-d} ; \gamma, c\right)=1-\exp \left(-\left(\gamma\left(T V_{t-d}-c\right)^{2}\right)\right), \gamma>0
\end{aligned}
$$

La función logística ${ }^{22}$ permite distinguir dos regímenes, que reciben la denominación de alto y bajo. El régimen alto ocurre cuando $\left(T V_{t-d}-c\right)$ es grande y positivo, ya que $\exp \left(-\gamma\left(T V_{t-d}-c\right)\right)$ tiende a cero $y$, por tanto, la expresión (17) tiende a 1 . Por el contrario, es bajo cuando $\left(T V_{t-d}-c\right)$ es grande y negativo, pues $\exp \left(-\gamma\left(T V_{t-d}-c\right)\right) \rightarrow \infty$ y $F\left(T V_{t-d} ; \gamma, c\right) \rightarrow 0 .^{23}$ La forma logística es válida cuando se cree que la transición ocurre de manera monotónica creciente.

En contraste, la función exponencial es de utilidad cuando importa el valor de la desviación absoluta de la variable de transición respecto al parámetro $c$.

Esta especificación, conocida como ESTAR (exponential smooth transition autoregressive model), restringe la dinámica de la ecuación a ser la misma siempre que $F\left(T V_{t-d} ; \gamma, c\right)$ adopte valores extremos. Así, las asimetrías entre regímenes estarán dadas por la magnitud absoluta de las desviaciones más que por el signo que presenten las mismas. Algunas aplicaciones relevantes de modelos ESTAR son el estudio del desalineamiento del tipo de cambio real respecto su valor fundamental dado por la paridad de poder adquisitivo (Michael et al., 1997; Taylor et al., 2001; o Chen y Wu, 2000); el ajuste no lineal de desviaciones de la paridad no cubierta de intereses (Sarno et al., 2006); o la reversión no lineal a la media de los futuros de acciones (Monoyios y Sarno, 2002).

\footnotetext{
${ }^{22}$ Al emplear la función (17) se obtendrá un modelo regresivo con transición suave logística (LSTAR, por su sigla en inglés).

${ }^{23}$ Dado que la función de transición puede adoptar un continuo de valores entre cero y uno, la caracterización de un modelo STAR con sólo dos regímenes puede resultar arbitraria sobre todo cuando el valor del parámetro de suavización es bajo y, por tanto, hay muchos valores por fuera de los extremos. En este sentido, van Dick et al. (2002) plantean que puede pensarse a un modelo STAR como una forma de permitir un "continúo" de regímenes.
} 
Las extensiones de los STAR a contextos multivariados (modelos VAR o sistemas de ecuaciones) han sido estudiadas, entre otos, por Weise (1999), van Dijk (2001), Camacho (2004) y Mendoza (2004). La forma general de un modelo con ajuste no lineal al equilibrio es:

$$
\begin{aligned}
& \Delta Y_{t}=\Pi_{1,0}+\Gamma D_{t}+\alpha_{1} M_{t-1}+\sum_{j=1}^{p} \Pi_{l, j} \Delta Y_{t-j}+ \\
& \left(\Pi_{2,0}+\alpha_{2} M_{t-1}+\sum_{j=1}^{p} \Pi_{2, j} \Delta Y_{t-j}\right) F\left(T V_{t-d} ; \gamma, c\right)+\varepsilon_{t}
\end{aligned}
$$

Donde $\Delta Y_{t}$ es un vector $(\mathrm{nx} 1): \Delta y_{1}, \Delta y_{2}, \ldots, \Delta y_{n}, D_{t}$ es un vector de dummies (mx1) que controlan los valores atípicos y $\Gamma(\mathrm{m} \times \mathrm{n})$ es su respectiva matriz de coeficientes. Asimismo, $\Pi_{1,0}$ y $\Pi_{2,0}$ son vectores ( $\left.\mathrm{n} \times 1\right)$ que contienen las constantes de la parte lineal y no lineal en cada caso. Las matrices $\Pi_{1, j}$ y $\Pi_{2, j}$ para $j: 1, \ldots, p$ y de dimensión (nxn) contienen los coeficientes de la parte autorregresiva en cada sección del modelo. Por su parte, $\alpha_{1}$ y $\alpha_{2}$ son matrices (nxr) con los coeficientes de ajuste de los desvíos respecto a las relaciones de largo plazo, donde $r$ indica el número de ecuaciones de cointegración. Dado que $\alpha$ se encuentra en el componente lineal y en el no lineal de (19), esta especificación tiene como posibilidad la transición suave en el mecanismo de corrección de errores. Justamente, el vector de corrección al equilibrio se denota por $M_{t}=\beta^{\prime} X_{t}$ de dimensión (rx1), $\beta$ es una matriz (nxr) con los coeficientes de la relación de largo plazo y $X_{t}(\mathrm{n} \times 1)$ representa los niveles de las variables incluidas en el modelo.

En cuanto a los pasos operativos necesarios para implementar un modelo STAR, Teräsvirta (1994) propone un procedimiento para el caso de univariado, Granger y Teräsvirta (1993) examinan el multivariado y Camacho (2004), entre otros, lo extiende a un contexto multiecuacional. El proceso guarda muchas similitudes en cualquiera de sus variantes y consta, sintéticamente, de cuatro etapas: i) estimación del modelo lineal; ii) prueba de no linealidad; iii) estimación no lineal; y iv) cómputo de las funciones de impulso-respuesta.

La primera etapa consiste en estimar un modelo lineal que actuará como base para contrastar la hipótesis alternativa de no linealidad. La estimación del mismo sigue las técnicas habituales de series de tiempo. Al respecto, es importante controlar por valores atípicos y obtener residuos con buen comportamiento (sin autocorrelación ni heterocedasticidad).

La segunda etapa es la prueba de linealidad. Cuando la hipótesis alternativa a la linealidad es la transición suave exponencial (modelo ESTAR), ${ }^{24}$ Teräsvirta (1994) sugiere emplear una expansión de Taylor de primer orden $\left(T_{l}^{e}\right)$ para obtener una regresión auxiliar que servirá de base para el contraste. Para ello, se debe tomar (18) y evaluar $T_{l}^{e}$ en $\gamma=0$. Luego de varias simplificaciones se obtendrá la siguiente regresión auxiliar (en notación vectorial):

\footnotetext{
${ }^{24}$ La regresión auxiliar se modifica cuando la especificación se realiza vía la función logística (modelo LSTAR). También existen pruebas generalizadas que son válidas tanto cuando se desconoce (o no se tiene una intuición a priori) si la alternativa relevante es la expresión (17) o la (18). Al respecto, véase Luukkonen et al. (1998).
} 


$$
Y_{t}=\beta_{0}^{\prime} X_{t}+\beta_{1}^{\prime} X_{t} T V_{t-d}+\beta_{2}^{\prime} X_{t} T V_{t-d}^{2}+\omega_{t}
$$

En la cual, $X_{t}=\left[1, y_{t-1}, y_{t-2}, \ldots, y_{t-d}\right] ; \beta_{0}=\left\lfloor\beta_{0,0}, \beta_{0,1}, \ldots, \beta_{0, p}\right\rfloor ; \quad \beta_{1}=\left\lfloor\beta_{1,0}, \beta_{1,1}, \ldots, \beta_{1, p}\right\rfloor ; \quad$ y $\beta_{2}=\left\lfloor\beta_{2,0}, \beta_{2,1}, \ldots, \beta_{2, p}\right\rfloor$. En (20), probar la hipótesis de linealidad $\gamma=0$ es equivalente a probar que $\beta_{1}=0$ y $\beta_{2}=0$.

Según Teräsvirta (1994), el procedimiento recomendado consiste en una prueba $F$ con la siguiente secuencia: i) estimar el modelo bajo la hipótesis de linealidad y computar la suma de residuos al cuadrado $\left(R S S_{0}\right)$; ii) estimar la regresión auxiliar (20) y obtener $R S S_{1}$; y iii) computar el valor crítico del estadístico:

$$
L M=\frac{\left(R S S_{0}-R S S_{1}\right) / l_{1}}{R S S_{0} / l_{2}}
$$

Los grados de libertad $l_{1}$ se calculan como la diferencia entre el número de parámetros del modelo irrestricto y el restricto; mientras que $l_{2}$ se calcula como el número de observaciones menos los parámetros del modelo irrestricto. En sistemas o modelos VAR, la práctica recomendada es evaluar con una prueba $F$ la hipótesis en cada una de las ecuaciones. Asimismo, Weise (1999) propone utilizar un contraste de razón de verosimilitud para evaluar la hipótesis $H_{0}: \gamma=0$ en todas las ecuaciones, contra $H a: \gamma \neq 1$ en al menos alguna de éstas. Cuando la prueba de linealidad se efectúa sobre una lista de posibles variables de transición, el resultado de la misma es útil para definir qué variable será la efectivamente considerada. En tal caso se selecciona la variable $T V$ con el estadístico $L M$ más alto.

El tercer paso en la especificación del modelo STAR es su estimación, que puede hacerse por cualquier método convencional no lineal, lo que se requerirá entonces definir condiciones iniciales. Si éstas son apropiadas será más probable alcanzar un máximo en la función de verosimilitud.

Para encontrar tales condiciones de inicialización se sugiere realizar una búsqueda de grilla de dos dimensiones (two dimensional grid search) sobre los parámetros $\gamma$ y $c$. En este sentido, nótese que una vez que el valor de ambos coeficientes está fijo, la función $F\left(T V_{t-d} ; \gamma, c\right)$ se encontrará en el intervalo $[0,1]$ en cada observación y ecuaciones como la (12) o la (19) serán lineales en todos sus argumentos. En la búsqueda de grilla se iteran distintos valores de $\gamma$ y $c$ tomados a intervalos que sean relevantes según sus respectivas escalas de variabilidad. La estimación condicional puede hacerse por un método lineal como OLS o SUR. Los parámetros del modelo lineal que surjan de la combinación de $\gamma$ y $c$ que maximiza la función de verosimilitud (o minimiza el determinante de la matriz de covarianzas de los residuos) serán el punto de partida para estimar el modelo STAR irrestricto.

A menudo la teoría también proporciona algún condicionamiento sobre el parámetro de suavización $(\gamma)$ o sobre el umbral $(c)$. En aquellos modelos donde el desalineamiento pasado es la variable de estado $\left(T V_{t-d}=M_{t-d}\right)$, suele imponerse la condición $c=0$. La razón es simple, si esta brecha tiende a cero, entonces, $F\left(T V_{t-d} ; \gamma, c=0\right)=1-\exp \left(-\left(\gamma\left(M_{t-d}\right)^{2}\right)\right) \rightarrow 0$, lo 
que nos ubica en uno de los regímenes extremos. En los trabajos sobre paridad de poder adquisitivo, los costos de transacción y trasporte imponen límites al arbitraje y, así, desalineamientos pequeños se interpretan como un estado donde la PPA no opera, o no lo hace plenamente. El corolario será que el tipo de cambio se comporta como un random walk si $M_{t-d}$ es bajo.

En el modelo teórico aquí evaluado $F\left(M_{t-d} ; \gamma, c=0\right) \rightarrow 0$ significa que se reducirá la incidencia de las expectativas de los traders fundamentalistas y que la trayectoria de precios podría incluso ser divergente de acuerdo a lo expuesto en la segunda sección.

Por último, una herramienta útil en un modelo STAR sólo aplicado a sistemas de ecuaciones es el cómputo de funciones de impulso-respuesta generalizadas.

Describir metodológicamente las mismas escapa por mucho al alcance de este trabajo. La idea intuitiva es que en un modelo STAR el efecto de los shocks depende de la historia, del tamaño y del signo de las perturbaciones. No es lo mismo un choque dado un desalineamiento inicial pequeño respecto a uno alto (dependencia de estado); así como tampoco es irrelevante el tamaño del shock pues implica distintas trayectorias dinámicas futuras de las variables endógenas. Tales características no pueden ser capturadas por los métodos tradicionales para calcular funciones de impulso-respuesta (Mendoza, 2004). La alternativa consiste en la generalización del concepto a modelos no lineales por métodos de Montecarlo o bootstrapping. En este trabajo se ha empleado la propuesta de bootstrapping de Koop et al. (1996) y se remite al lector interesado a dicha referencia para una discusión de los detalles técnicos.

\section{No linealidad en el ajuste de los precios de los commodities: resultados empíricos}

A efectos de organizar la exposición del modelo empírico se ha dividido esta sección en cuatro partes. En la primera de ellas, se presentan las variables y se describen las fuentes de datos utilizadas. Posteriormente, se discute la estimación del equilibrio de largo plazo de los precios de los commodities y se estudia la evolución temporal del desalineamiento. En la tercera sección se exponen y evalúan los resultados de la prueba de no linealidad, la interpretación de la función de transición y sus regímenes y la estimación del sistema. Para finalizar se efectúa un análisis de impulso-respuesta donde se investiga la reacción a corto plazo de los precios de los commodities frente a choques en sus determinantes bajo distintos regímenes, que dependen del grado de influencia de los esquemas de formación de expectativas de chartistas y fundamentalistas.

\subsection{Las variables del modelo empírico}

El primer dilema a resolver es definir cuáles precios de commodities se buscarán explicar. Por tratarse el presente de un estudio con un nivel elevado de generalidad y siendo que, en principio, la teoría revisada es representativa de un conjunto amplio de bienes, se juzgó pertinente elaborar un índice agregado. En esto no hay discrepancias sustanciales con otros trabajos sobre determinantes de los precios. La única omisión en cuanto a los componentes del índice han sido los commodities energéticos, particularmente el petróleo. Autores como Beenstock (1988) o, más recientemente, Cheung y Morin (2007) han destacado que el petróleo tiene una dinámica propia con baja conexión respecto al resto de los commodities y que, por tanto, resulta conveniente modelarlo de manera aislada. Como los modelos STAR son 
demandantes en cuanto al número de observaciones necesarias para el testeo y estimación, se recurrió a datos de frecuencia mensual.

Así, empleando como fuente el International Finacial Statistics (IFS) se construyó una serie de precios de los commodities en términos reales (denotados por $P$ ) como promedio simple de cuatro grupos de bienes relevados por el FMl: alimentos, bebidas, materias primas de la agricultura y metales. Como deflactor se empleó el índice de precios al consumidor de los Estados Unidos (CPI). En el Gráfico 1 se ilustra la evolución del índice desde 1970:1 a 2007:7. ${ }^{25}$

\section{Gráfico 1. Índice de precios de los commodities en términos reales (escala logarítmica)}

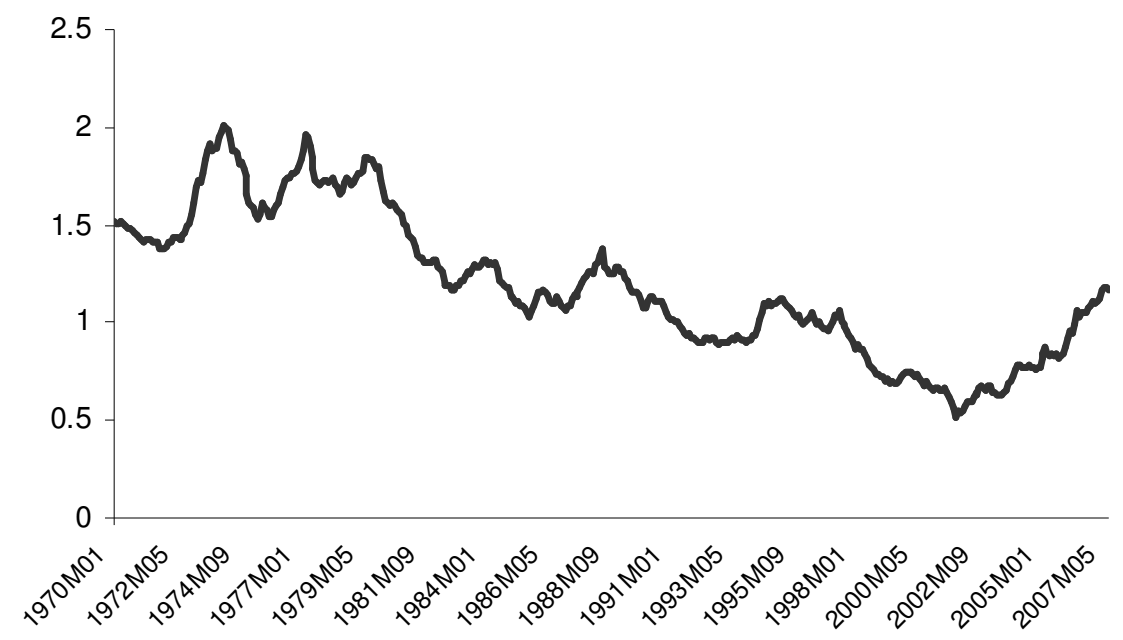

En términos reales los precios han descendido respecto a los máximos alcanzados a fines de la década del setenta. Lo hicieron de manera bastante marcada hasta el año 2001 donde alcanzaron su mínimo. El ascenso desde esa fecha hasta julio de 2007 ha sido muy importante (130\% aproximadamente), pero el nivel está todavía alejado de los picos anteriores.

En cuanto a los fundamentales de los precios se tiene, en primer término, al tipo de cambio real $(T C R)$ de Estados Unidos. La versión utilizada es la multilateral publicada periódicamente por la Federal Reserve Bank of New York. La variable está definida de manera tal que sube cuando el dólar se aprecia frente a las monedas de los principales socios comerciales.

Por otro lado, las condiciones monetarias se aproximaron, al igual que en Bastourre et al. (2008), vía una variable que mide la liquidez global ( $L I Q)$ y que se construye como la suma de la base monetaria de Estados Unidos y las reservas internacionales de los restantes bancos centrales del mundo. La primera es una serie del Board of Governors of the Federal Reserve System. Las reservas totales se obtuvieron del International Financial Statistics del FMI. De la

\footnotetext{
${ }^{25}$ Aquí vale una aclaración respecto a la cuestión de los commodities como activos financieros. Una observación que podría hacerse respecto a la serie de precios empleada es que se construye con datos de precios spot y no de precios de derivados u otros instrumentos que son los verdaderos vehículos de inversión en lo estrictamente financiero. El argumento es que, sin embargo, la utilización del precio spot es un muy buen indicador del retorno financiero de los commodities. En este sentido, Gorton y Rouwenhorst (2004) muestran que el retorno en una posición de futuros y el precio corriente tienen una correlación cercana a la unidad.
} 
misma fuente también se tomó el deflactor de precios implícito del PIB de los EE.UU. para expresar la liquidez en términos reales.

La variable para cuantificar el impacto de la demanda es sin duda la más compleja, pues es dificultoso medir la evolución del producto (o el ingreso) mundial con una frecuencia mensual.

La producción industrial ( $P R O$ ) es la alternativa seguida por todos los estudios en la materia cuando existe tal restricción de frecuencia. Hay que considerar sin embargo, que quienes poseen datos sistemáticos y confiables para esa variable son mayoritariamente países desarrollados. Emplear sólo éstos implicaría excluir economías emergentes de gran tamaño, particularmente China, cuya demanda es indicada como causante importante de las subas recientes. Atendiendo a estas consideraciones, se procedió de la siguiente manera. Se tomaron los índices de los países industriales a los que se adicionaron Corea, India, Malasia y China. Para ponderar a cada país en un índice se tomó la participación de cada país en el valor agregado industrial (VAl) cuya fuente es el Banco Mundial. ${ }^{26}$ Dado que para China no se dispone de una serie de producción industrial, se utilizó el dato anual del VAl y se lo menzualizó con una interpolación lineal.

Se incluyó también un índice real de la evolución de las acciones. La razón para incluir esta variable no proviene estrictamente de los antecedentes teóricos revisados en la primera sección, sino que obedece a una lógica distinta. En efecto, frente a la financiarización de los commodities, es necesario controlar por el rendimiento de activos alternativos para tomar en cuenta posibles sustituciones o complementariedades. Gorton y Rouwenhorst (2004) y Deutsche Bank (2005) encuentran que la correlación no condicional entre commodities y acciones es negativa y significativa en el largo plazo. Para Domanski y Healt (2007), la proporción neta de contratos largos de los agentes no comerciales está negativamente correlacionada, aunque tenuemente, con los índices bursátiles. Se consideró el índice Dow Jones deflactado por el CPI de los Estados Unidos ( $D O W)$.

Finalmente, las series de producción industrial y de liquidez internacional obtenidas fueron desestacionalizadas por el método X-12 Arima. El resto de las variables no evidenciaban un claro patrón estacional. Todas ellas se encuentran expresadas en logaritmos y resultaron no estacionarias de acuerdo a los tests habituales de raíces unitarias. ${ }^{27}$

\subsection{La estructura del modelo, la estimación del equilibrio y el desalineamiento}

El sistema propuesto es un caso híbrido. No se trata de un VAR simétrico con transición suave, como el presentado por Weise (1999), ya que sólo se permite, a partir de las restricciones teóricas, la presencia de una función de transición en la ecuación de precios de los commodities. Una alternativa podría haber sido emplear un ESTAR uniecuacional con dichos precios, pero eso hubiese implicado abandonar la posibilidad de estudiar la respuesta de corto

${ }^{26}$ El ponderador así obtenido se incrementó un 50\% en el caso de los países emergentes por dos razones. La primera es que las economías emergentes se encuentran subrepresentadas en la muestra. La segunda razón es que estos países tienen una mayor intensidad de consumo de commodities por unidad de producto. Capturar específicamente una cuantía de aumento para el coeficiente escapa a las posibilidades de este estudio; pero incrementarlo parece una mejor alternativa que ignorar los dos efectos antes mencionados. La serie así obtenida no altera significativamente la dinámica de la producción industrial.

${ }^{27}$ Los resultados de estas pruebas se encuentran disponibles a disposición de quien los solicite. 
plazo de los commodities frente a shocks en los fundamentales. En concreto, tomando (19) como referencia se propone trabajar con el siguiente sistema:

$$
\begin{aligned}
& \Delta P_{t}=\beta_{11}+\Gamma_{11} D+\alpha_{11}\left(M_{t-1}\right)+\sum_{j=1}^{p} \Pi_{11, j} \Delta X_{t-p}+ \\
& \left(\beta_{12}+\Gamma_{12} D+\alpha_{12}\left(M_{t-1}\right)+\sum_{j=1}^{p} \Pi_{12, j} \Delta X_{t-p}\right)\left(1-\exp \left(-\left(\gamma\left(M_{t-d}\right)^{2}\right)\right)\right)+\varepsilon_{t}^{P} \\
& \Delta T C R_{t}=\beta_{2}+\Gamma_{2} D+\alpha_{2}\left(M_{t-1}\right)+\sum_{j=1}^{p} \Pi_{2, j} \Delta X_{t-p}+\varepsilon_{t}^{T C R} \\
& \Delta L I Q_{t}=\beta_{3}+\Gamma_{3} D+\alpha_{3}\left(M_{t-1}\right)+\sum_{j=1}^{p} \Pi_{3, j} \Delta X_{t-p}+\varepsilon_{t}^{L I Q} \\
& \Delta P R O_{t}=\beta_{4}+\Gamma_{4} D+\alpha_{4}\left(M_{t-1}\right)+\sum_{j=1}^{p} \Pi_{4, j} \Delta X_{t-p}+\varepsilon_{t}^{P R O} \\
& \Delta D O W_{t}=\beta_{5}+\Gamma_{5} D+\alpha_{5}\left(M_{t-1}\right)+\sum_{j=1}^{p} \Pi_{5, j} \Delta X_{t-p}+\varepsilon_{t}^{D O W}
\end{aligned}
$$

Donde, $X=[P, T C R, L I Q, P R O, D O W]$ y se igualó a cero el coeficiente indicativo del umbral $(c)$ por las razones expuestas previamente. Como antes, $M_{t}=P_{t}-F\left(X_{t}\right)$. Es claro que el principal foco de atención corresponde a la expresión (22) que contiene la dinámica de precios. Particular interés revisten los coeficientes de ajuste a la relación de largo plazo $\alpha_{11}$ y $\alpha_{12}$ correspondientes a cada régimen. Dada la estructura de shocks de la forma reducida, incluyendo ciertos supuestos de identificación necesarios para obtener shocks estructurales y conociendo los vectores de coeficientes $\Pi$, se obtendrán las respuestas dinámicas de corto plazo de la ecuación de precios.

Por el momento, no obstante, corresponde cuantificar el equilibrio dado los fundamentales para luego computar el delineamiento $M_{t}$. La lógica de estimación para el sistema (22)-(26) guarda similitudes con la metodología de Engle y Granger. Dado el orden unitario de integración de las series se plantea la posibilidad de una estimación consistente del largo plazo por mínimos cuadrados ordinarios (OLS). Sin embargo, este estimador tiene una distribución no normal cuando las series son integradas de orden uno y la inferencia basada en sus estadísticos- $t$ puede conducir a conclusiones erróneas.

Una alternativa para superar este inconveniente es emplear mínimos cuadrados ordinarios dinámicos (DOLS). Se trata de una modificación de la regresión OLS de manera que incluya valores pasados, presentes y futuros de los cambios en las variables del lado derecho de la ecuación (Stock y Watson, 1993). Si los errores estándar se corrigen además por heterocedasticidad y autocorrelación, entonces la inferencia será válida. Tal fue el procedimiento seguido considerándose, doce rezagos y doce adelantos para todas las variables del modelo (excluyendo los precios). 
En ambas especificaciones se incluyó una tendencia temporal, para controlar el posible deterioro de los precios subrayado por la tesis Prebisch-Singer aunque, en esta oportunidad, condicionado a la evolución de otros determinantes. En la Tabla 1 se presentan los principales resultados.

Tabla 1. Equilibrio de largo plazo por OLS y DOLS

\begin{tabular}{|c|c|c|}
\hline & & \\
\hline & OLS & DOLS \\
\hline Tipo de Cambio Real & $-0,4844$ & $\begin{array}{c}-0,4406^{\star * \star} \\
(0,0548)\end{array}$ \\
\hline Liquidez internacional & 0,4186 & $\begin{array}{c}0,4409^{\star * *} \\
(0,0619)\end{array}$ \\
\hline Producción Industrial & 1,7622 & $\begin{array}{c}0,8729^{\star * *} \\
(0,1977)\end{array}$ \\
\hline Dow Jones & $-0,1549$ & $\begin{array}{c}-0,0717^{\star * *} \\
(0,0145)\end{array}$ \\
\hline Constante & $-12,122$ & $\begin{array}{c}-9,4951^{\star * *} \\
(1,0613)\end{array}$ \\
\hline Tendencia Temporal & $-0,0072$ & $\begin{array}{c}-0,0062^{* * *} \\
(0,0002) \\
\end{array}$ \\
\hline $\mathrm{R}^{2}$ & 0,95 & 0,99 \\
\hline Test F de significatividad conjunta (P-valor) & - & 0,00 \\
\hline
\end{tabular}

Nota: errores estándar entre paréntesis, el símbolo *** indica que la variable es significativa al 1\%

Los signos de las variables se encuentran alineados con lo esperado en términos teóricos, según lo que se desprende de ambos modelos. Se han omitido los errores estándar en la columna OLS por los problemas mencionados anteriormente. Para obtener conclusiones sobre la significatividad estadística la referencia será el estadístico $t$ asociado al DOLS.

Respecto al valor de los coeficientes, se obtiene en primer lugar que una devaluación real (caída) del dólar de un $1 \%$ implica un $0,48 \%$ de aumento de los commodities según la estimación por OLS $(0,44 \%$ según la columna del DOLS).

La liquidez internacional, a su turno, muestra una elasticidad similar (en valor absoluto). Esto es compatible con la visión de Frankel (2006), para quien la explicación de los altos precios actuales de los commodities debe buscarse en la actitud condescendiente de los bancos centrales respecto a la expansión de los medios de pago.

Los coeficientes de la producción industrial y del Dow Jones son los que deparan mayores discrepancias cuando se cambia la metodología. En la columna OLS la elasticidad de la variable $P R O$ es 1,76 con lo cual el precio de los commodities responde más que proporcionalmente a un desplazamiento en la demanda. La elasticidad ingreso se encuentra cercana a la unidad $(0,87)$ si se aplica DOLS.

El rendimiento de las acciones impacta negativamente en la cotización de los commodities. En ambas estimaciones se obtienen resultados sugerentes de una moderada significatividad económica; aunque la significatividad estadística es clara. En línea con lo encontrado por Domanski y Healt (2007), prima el efecto sustitución entre ambas clases de activos. 
También es relevante el efecto de la tendencia temporal, cuyo coeficiente es similar en las dos estimaciones. El hecho era esperable considerando la simple inspección visual del Gráfico 1. Otro aspecto a destacar es que el ajuste del modelo es elevado de acuerdo a la medida del $R^{2}$, lo que lleva a intuir que la brecha entre el precio corriente y el precio fundamental debería tener un rango de variabilidad relativamente acotado.

En síntesis, los resultados de largo plazo son consistentes con lo predicho por las ideas teóricas. Con esta información es posible construir ahora dos series de precios de "equilibrio" correspondientes a cada estimador. A continuación se grafican las mismas junto con la serie de precios corrientes.

\section{Gráfico 2. Índice de precios de los commodities y estimaciones del equilibrio (escala logarítmica)}

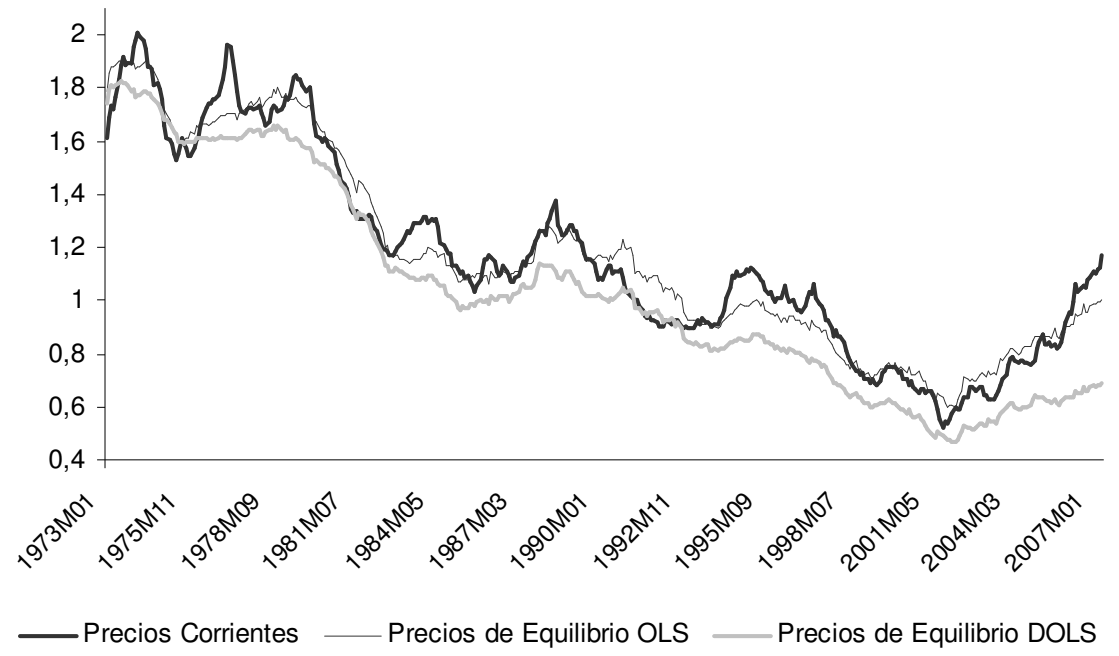

Como se puede verificar en el Gráfico 2, la serie correspondiente al precio de equilibrio OLS se encuentra sistemáticamente por encima de la obtenida por DOLS y corta mucho más a menudo la línea de precios corrientes. Esto se explica por el hecho de que en el cálculo con los fundamentales en el DOLS se ha omitido el efecto de las diferencias de las variables explicativas, sus adelantos y rezagos; con lo cual el desalineamiento no tiene necesariamente una media de cero por construcción.

Atendiendo este último hecho, y dado que los coeficientes son consistentemente estimados por OLS, se tomará su equilibrio respectivo para calcular de misalignment $\left(M_{t}\right)$. Este tendrá un doble rol: como variable de ajuste de las desviaciones a corto plazo; y como potencial determinante del estado y la transición. Seguidamente se ilustra su evolución en el tiempo. 


\section{Gráfico 3. Desalineamiento del precio de los commodities respecto a sus fundamentales}

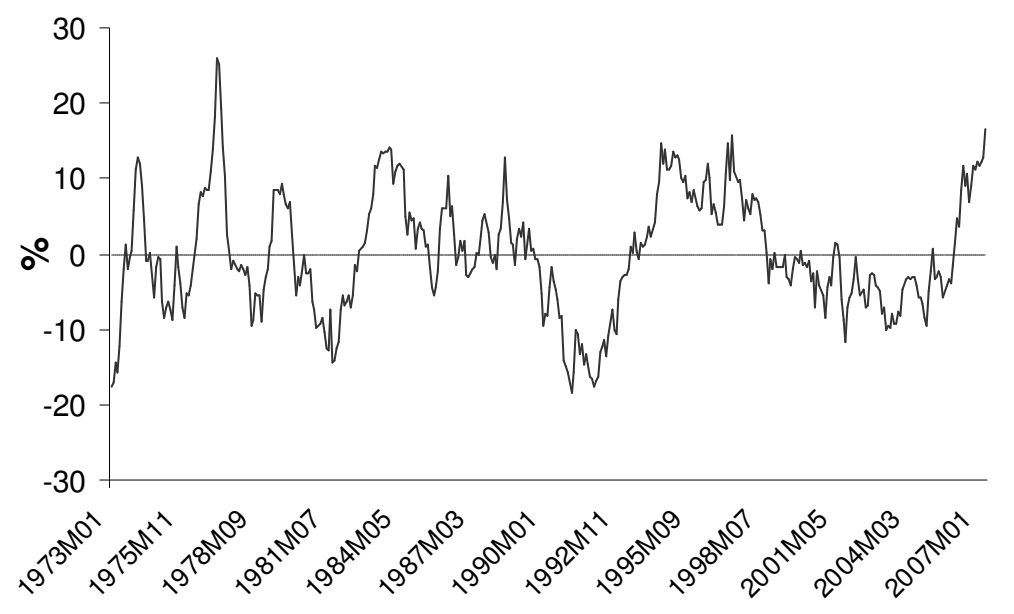

Temporalmente pueden ubicarse dos máximos en el desalineamiento, uno a fines de la década del setenta y otro en junio de 2007, último dato con el que se cuenta con información para todos los fundamentales. Por su parte, la brecha entre el nivel observado y el equilibrio fue negativa y alta en valor absoluto durante los primeros meses de la muestra, entre los años 1981-1982 y a comienzos de la década del noventa.

Muy interesante resulta analizar la última etapa de altos precios de los commodities con esta información. En efecto, el Gráfico 2 sugiere que cambios en variables fundamentales como la depreciación del dólar, el aumento de la demanda de commodities o la mayor liquidez mundial han ocasionado un fuerte incremento en los precios de equilibrio. Sin embargo, la suba efectivamente observada en los mismos ha sido incluso más pronunciada (Gráfico 3). Esto produjo un pico de desalineamiento positivo, sólo comparable con el máximo alcanzado en las cercanías al segundo gran shock en los precios del petróleo.

De confirmarse que el desalineamiento es una variable relevante para determinar la forma del ajuste en la dinámica de precios, habrá cierto espacio para sostener que la presencia de inversores financieros también merece un lugar relevante en la explicación de las tendencias actuales en los mercados de los commodities.

\subsection{El modelo de corto plazo y la dinámica no lineal de ajuste al equilibrio}

Corresponde en primer lugar construir un modelo lineal, que actúe como referencia para evaluar la hipótesis alternativa de no linealidad. Con el equilibrio calculado por OLS se procedió entonces a estimar un modelo autorregresivo vectorial de corrección al equilibrio (VECM) simétrico, con las diferencias de las cinco variables en consideración.

Para ello, se efectuó un control por valores atípicos a partir de la inspección visual de las series en diferencias, lo que implicó que se incluyeran 13 dummies puntuales. ${ }^{28}$

Luego de ello, se efectuó la selección de rezagos ( $p$ según la notación previa) para la parte en diferencias. Siguiendo a Granger y Teräsvirta (1993), se decidió adoptar como criterio de

28 Correspondientes a observaciones de los meses 1974:12, 1975:01, 1977:03, 1981:11, 1982:06, 1987:01, 1987:11, 1988:07, 1993:08, 1997:04, 2003:05, 2003:09 у 2006:08. 
selección el número de lags que arrojase residuos de buen comportamiento. Según dichos autores, esta práctica es preferible aun a riesgo de trabajar con un modelo sobreespecificado, para no afectar los resultados de la prueba de no linealidad con la presencia de correlación serial en los errores.

Partiendo de 3 rezagos, número sugerido por el criterio de información de Akaike, se incluyeron rezagos hasta que las pruebas de no autocorrelación y homocedasticidad sobre los residuos de las cinco ecuaciones llevaran al no rechazo de tales hipótesis nulas. Con $p=5$ se lograron estos objetivos. $^{29}$

Hay que subrayar que el modelo lineal así estimado presentó una ecuación para la dinámica de precios con un término de ajuste al equilibrio que fue negativo y significativo al $1 \%$. Específicamente, su valor fue -0,066.

Posteriormente, siguiendo los pasos operativos descritos en la sección metodológica, se realizó el contraste de linealidad sobre la ecuación de precios de los commodities. Esta prueba se efectuó a partir de la especificación genérica dada en (20). Una vez que se toman en cuenta las variables del modelo y se considera la restricción teórica de que el desalineamiento respecto al equilibrio actúe como variable de transición, la regresión auxiliar necesaria para la prueba adopta la siguiente forma:

$$
\begin{aligned}
& \Delta P_{t}=\beta_{0, p}^{\prime} \Delta X_{t-p}+\Gamma D+v_{1} M_{t-1}+\beta_{1, p}^{\prime} \Delta X_{t-p} M_{t-d}+v_{2} M_{t-l} M_{t-d}+ \\
& \beta_{2, p}^{\prime} \Delta X_{t-p} M_{t-d}^{2}+v_{3} M_{t-1} M_{t-d}^{2}+\omega_{t}
\end{aligned}
$$

En la que $\Delta X=\left\lfloor 1, \Delta P_{t-p}, \Delta T C R_{t-p}, \Delta L I Q_{t-p}, \Delta P R O_{t-p}, \Delta D O W_{t-p}\right\rfloor$ y $p: 1, \ldots, 5$. Nótese que los primeros tres términos en (27) corresponden a la especificación lineal de la ecuación para el cambio en el precio de los commodities en el VECM que se describió anteriormente. En la Tabla 2 se exponen los resultados del test. ${ }^{30}$ Al respecto, se tomaron 11 posibles variables de transición a partir de $M_{t-d}$, considerando los rezagos $d: 2, \ldots, 12 .^{31}$ En las columnas se tabulan tanto el valor del estadístico $L M$ calculado según (21) como el valor-p del test $F$ asociado al mismo. Recuérdese que la hipótesis nula es la de linealidad en la ecuación del cambio en los precios de los commodities.

\footnotetext{
${ }^{29}$ Se omiten la presentación de los resultados respectivos para facilitar la exposición, pero se ponen a disposición del lector interesado.

${ }^{30}$ Este test fue programado en E-views. El código se encuentra disponible previa solicitud al autor.

${ }^{31}$ No se incluyó el primer rezago en el desalineamiento por razones conceptuales, asociadas al modelo de agentes heterogéneos. La idea es que existen costos en términos de tiempo, información y análisis para procesar la información. Para captar parcialmente este tipo de efecto, se impuso que la variable de estado tenga un rezago mayor a $M_{t-1}$ que es información propia del modelo.
} 
Tabla 2. Resultados del test de linealidad

\begin{tabular}{l|c|c}
\hline Variable de Transición & Estadístico $L M$ & Test $F$ (valor-p) \\
\hline \hline$M_{t-2}$ & 1,6345 & 0,0060 \\
\hline$M_{t-3}$ & 1,3463 & 0,0661 \\
\hline$M_{t-4}$ & 1,1706 & 0,2093 \\
\hline$M_{t-5}$ & 1,2590 & 0,1213 \\
\hline$M_{t-6}$ & 1,2532 & 0,1260 \\
\hline$M_{t-7}$ & 1,1620 & 0,2199 \\
\hline$M_{t-8}$ & 1,3013 & 0,0911 \\
\hline$M_{t-9}$ & 1,3291 & 0,0749 \\
\hline$M_{t-10}$ & 1,3057 & 0,0884 \\
\hline$M_{t-11}$ & 1,4904 & 0,0213 \\
\hline$M_{t-12}$ & 1,5735 & 0,0104 \\
\hline
\end{tabular}

Se desprende de la Tabla 2 que cuando se emplean los rezagos 2, 3, 8, 9, 10, 11 y 12 el test $F$ rechaza la hipótesis nula con un nivel de significatividad del 10\%. Al mismo tiempo, el máximo valor del estadístico $L M$ se da al utilizar $M_{t-2}$ como variable de transición siendo éste, además, el único caso en que se rechaza $H_{0}$ al 1\%. Es por ello que se seleccionó al desalineamiento con dos lags como variable de estado.

En la secuencia para la construcción del modelo ESTAR, el siguiente paso es la estimación del sistema no lineal, para lo cual deben proporcionarse condiciones iniciales para todos los coeficientes en las ecuaciones (22) a (26). Dichas condiciones pueden encontrase a partir de un sistema lineal una vez que se fija el valor del parámetro $\gamma$, como ya se mencionó previamente.

Se recurrió, a una restricción teórica para dar un valor de inicialización a $\gamma$. Se tomaron como punto de partida las expresiones (5) y (6) con la idea de que una referencia inicial puede resultar aquella en la cual los administradores de cartera ponderan por igual las expectativas de los agentes fundamentalistas y de los chartistas. Esto significa que se ajustó $\gamma$ hasta que, dadas la función exponencial y la variable de transición $M_{t-2}$, se verificase en el período muestral considerado (1973:7 a 2007:4) que:

$$
\bar{w}_{t}=1-\exp \left(-\left(\gamma\left(M_{t-2}\right)^{2}\right)\right)=\frac{1}{2}
$$

Un valor $\gamma=251,9$ produce que en la muestra efectiva la función de transición $w_{t}$ adopte, en promedio, el valor buscado. A continuación se grafica tanto la relación entre $w_{t}$ y $M_{t-2}$ (lado izquierdo) como la evolución temporal de la función de exponencial (lado derecho) para $\gamma=251,9$. 
Gráfico 4. Función de transición y su evolución temporal $(\gamma=251,9)$
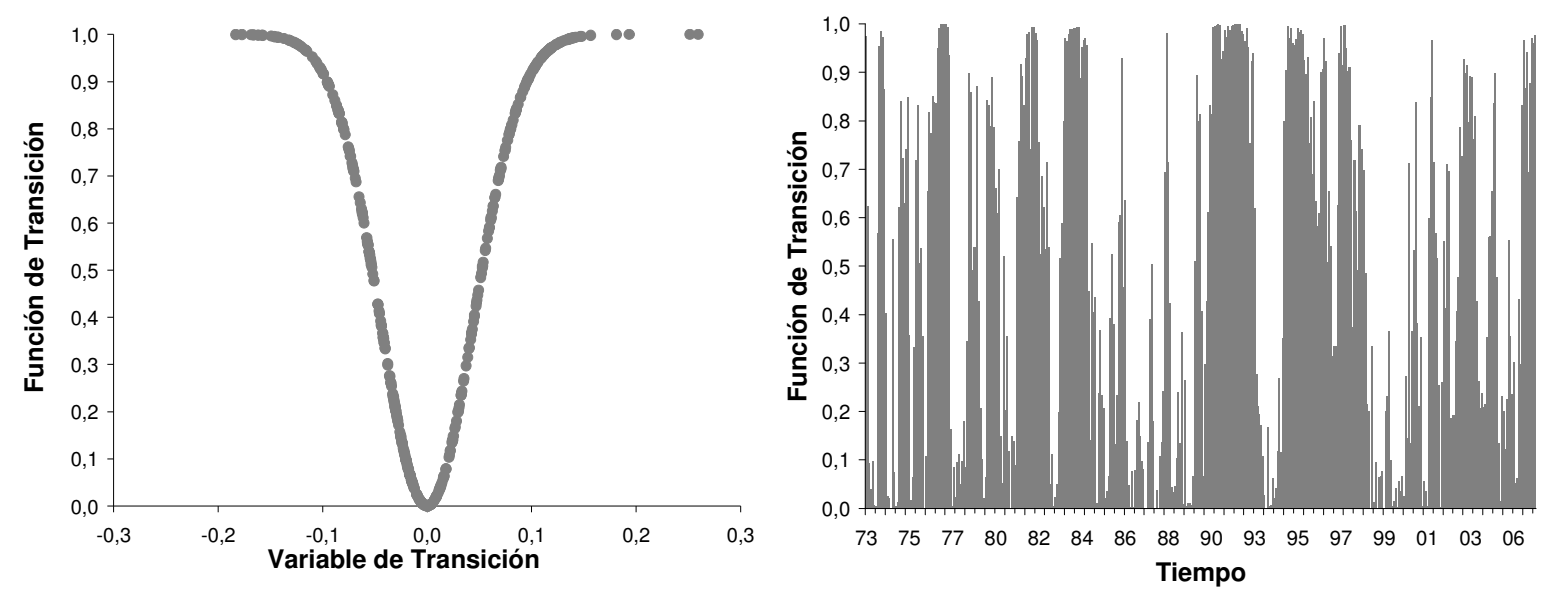

En ambas figuras puede verificarse rápidamente que la media de la función de transición se encuentra cercana a 0,5 . Del lado derecho, se observa como los distintos períodos temporales se asocian a valores altos o bajos de $w_{t}$. A medida que este sube, mayor debería ser la incidencia de las expectativas de corrección al equilibrio sobre los precios de los commodities. Esta hipótesis se podrá verificar una vez que estimado el modelo no lineal liberado, en el cual se maximiza la función de verosimilitud del sistema dadas las condiciones iniciales.

Con los parámetros fijados según el modelo lineal correspondiente a $\gamma=251,9$ se procedió entonces a estimar el sistema (22)-(26) con $M_{t-d}=M_{t-2}$ por SUR ${ }^{32}$.

A los efectos de evaluar la hipótesis central de este trabajo, el interés se centra en los coeficientes de ajuste a la relación de largo plazo $\alpha_{11}$ y $\alpha_{12}$ en la ecuación del cambio en los precios de los commodities.

Al respecto, se obtuvo en la estimación parámetro de ajuste de la parte lineal $\left(\alpha_{11}\right)$ con un valor de 0,2835 con un error estándar de 0,1722 , por lo que la variable es significativa al $10 \%$. Esto significa que si la función de transición $\left(w_{t}\right)$ valiese 0 , la dinámica de precios sería explosiva pues un desalineamiento inicial en vez de corregirse se amplificaría a una tasa del $28 \%$ mensual. La interpretación para dicho valor dentro de las líneas sostenidas en el modelo teórico puede pasar por el comportamiento de agentes que emplean herramientas de análisis técnico y que reaccionan extrapolando los niveles pasados de desalineamiento hacia el futuro.

Más allá de la interpretación de $\alpha_{11}$, es crucial notar que la estabilidad global de la ecuación de precios se encuentra garantizada. Esto ocurre porque el valor del coeficiente de ajuste de la parte no lineal $\left(\alpha_{12}\right)$ encontrado fue negativo y mayor a $\alpha_{11}$ en valor absoluto.

Concretamente, se obtuvo que $\alpha_{12}=-0,3636$, siendo la variable significativa al $5 \%$. Si la incidencia de los fundamentalistas es máxima $\left(w_{t}=1\right)$, el coeficiente de ajuste global al equilibrio será $\alpha_{11}+\alpha_{12}=0,2835-0,3636=-0,0801$. Así, cuando el impacto de las expectativas

${ }^{32}$ El código en E-Views empleado en esta parte también se encuentra disponible previo pedido al autor. 
de ajuste al equilibrio alcanza un pico, la brecha en los precios corrige a tal referencia a una tasa del 8\% mensual. En el Gráfico se 5 pone de manifiesto el impacto de la función de transición sobre la corrección del desalineamiento.

\section{Gráfico 5. Función de transición y ajuste al equilibrio en el modelo no lineal}

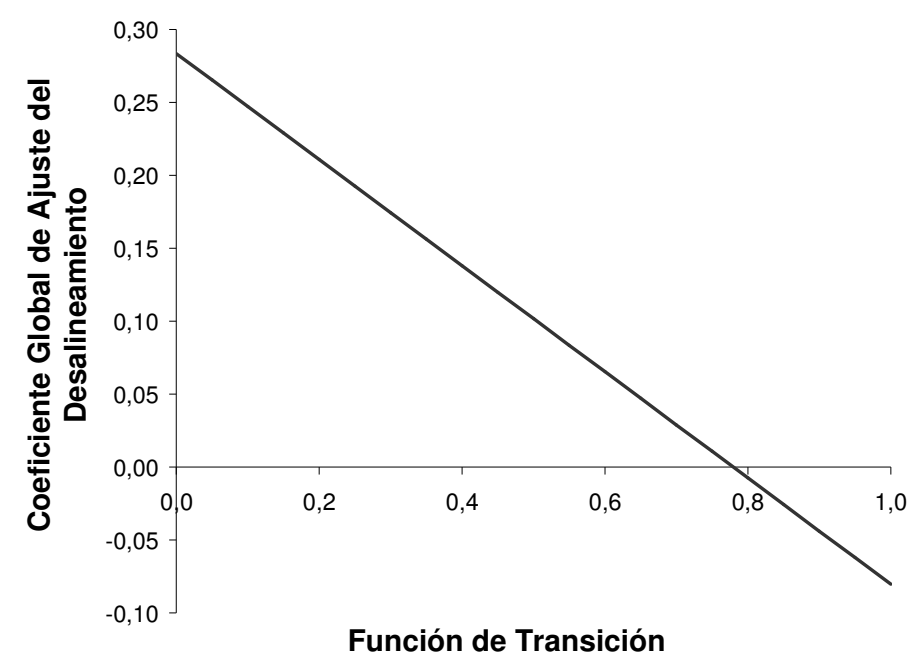

En cada observación para la cual $w_{t}>0,78$ el ajuste global de la brecha entre el precio corriente y el fundamental será negativo. El corolario es que altos misalignments en el pasado implican altos valores de la función de transición y esto marcará una tendencia de reversión futura.

Otro dato muy relevante para interpretar el sistema resultante es el parámetro de suavizamiento de la función exponencial. Este resultó significativo al 1\% y su valor estimado fue $\gamma=994,3$. En el Grafico 6 se exponen la función $w_{t}$ y su evolución en el tiempo.

Gráfico 6. Función de transición y su evolución temporal en el modelo liberado $(\gamma=994,3)$
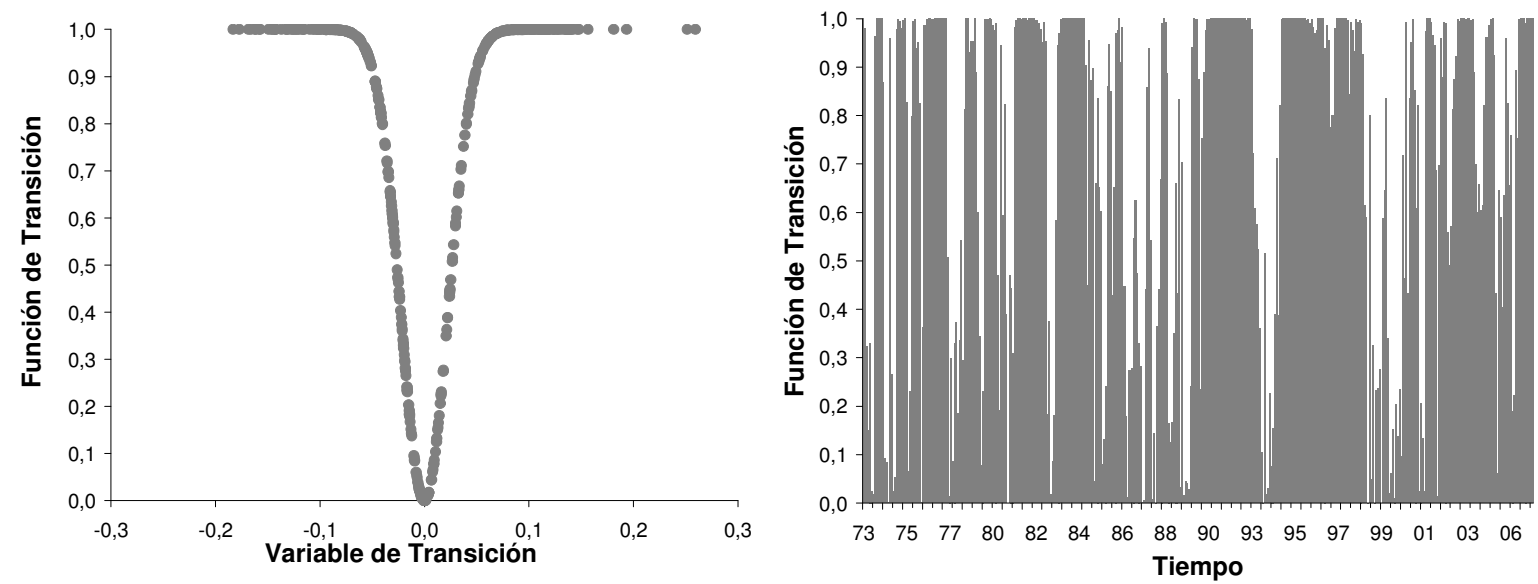

Se obtiene así una función de transición un tanto más abrupta que aquella que se empleó como condición de inicialización de la estimación. El promedio muestral del valor de $w_{t}$ es 0,715 . Si se desean interpretar los resultados en correspondencia con el modelo de agentes 
heterogéneos, podría decirse que el ajuste medio de los administradores de cartera dado en (5) sería el siguiente:

$$
E\left(\Delta P_{t+1}^{A}\right)=\left(1-\bar{w}_{t}\right) E\left(\Delta P_{t+1}^{C}\right)+\bar{w}_{t} E\left(\Delta P_{t+1}^{F}\right)=0,284 E\left(\Delta P_{t+1}^{C}\right)+0,716 E\left(\Delta P_{t+1}^{F}\right)
$$

En cuanto a la utilidad de estos resultados para el análisis del ciclo alcista actual, el modelo empírico estaría sugiriendo la idea de una cercanía a un punto de reversión hacia el equilibrio de largo plazo (lado derecho del Gráfico 6). Sin embargo, este movimiento no será necesariamente inmediato. Han existido en el pasado períodos de altos misalignments que perduraron aún más en el tiempo que el referido al último período dentro de la muestra.

Auque fuertemente indicativos de la relevancia de efectos no lineales en la dinámica de los precios de las commodities, se sugiere tomar estos resultados econométricos con una dosis de cautela.

En este sentido, una cuestión muy relevante a considerar es que es posible que el índice agregado esté acotando demasiado la volatilidad característica de los commodities. Esto pude explicar por qué el desalineamiento no alcanza proporciones elevadas. Por tratarse este trabajo de una primera aproximación general a la temática, la opción de una serie amplia de precios sigue estando justificada. No obstante, es de esperar que la potencia de la metodología se incremente de ser aplicada a grupos de bienes más semejantes o, más aún, a commodities específicos. Este tipo de extensión es una guía importante para futuras investigaciones.

\subsection{Dependencia de estado y efecto de los shocks en los fundamentales sobre los precios}

Además de brindar los valores de $\alpha_{11}, \alpha_{12}$ y $\gamma$, la estimación libre del sistema (22) a (26) arroja los valores de los coeficientes de los cambios de $X=[P, T C R, L I Q, P R O, D O W]$ en los distintos vectores $\Pi_{i, j}$. En los modelos VAR, es usual trabajar las interacciones dinámicas es a través de las funciones de impuso-respuesta. Como se adelantaba en la sección metodológica, la propuesta seguida aquí fue aplicar las funciones de impulso-respuesta generalizadas desarrolladas por Koop et al. (1996).

Sin entrar en los detalles técnicos, es importante notar que la simulación significó asignar cada observación a un régimen. Para ello, se tomó como punto de corte el valor 0,5 de la función de transición y se computaron dos regímenes: de desalineamiento alto (equivalente a $w_{t}>1 / 2$ ) y de desalineamiento bajo $\left(w_{t} \leq 1 / 2\right)$. Toda observación queda encuadrada entonces en alguno de estos dos estados. La selección de shocks correspondientes a los dos regímenes se basa en esta asignación temporal.

La segunda observación es que la simulación requiere que se proyecte hacia adelante el valor del desalineamiento para mantener actualizada la función de transición. Es decir, se debe indicar un valor a $w_{t}$ en cada período posterior al shock. Así, la diferencia entre simulaciones estará dada por la condición de partida de desalineamiento, pero este puede ir modificándose endógenamente con posterioridad a la perturbación. La forma de la secuencia es que el propio shock genera desalineamiento lo que, a su vez, ocasiona uno u otro tipo de ajuste inmediato dado el estado. Luego de ello el estado se modifica por el cambio en el desalineamiento. 
En los Gráficos (7) a (11) se muestran los resultados. La línea oscura es la respuesta estandarizada con un choque de un desvío estándar en la variable que sufre la perturbación. Las líneas en gris son las bandas de confianza del 10\%. En líneas punteadas se muestra el efecto de un choque equivalente a tres desvíos estándar (su respuesta está normalizada). Con esto último se intentan captar posibles no linealidades producidas por el tamaño de la perturbación.

\section{Gráfico 7. Respuesta dinámica acumulada de los precios de los commodities ante un} shock en la propia variable

Régimen de desalineamiento alto

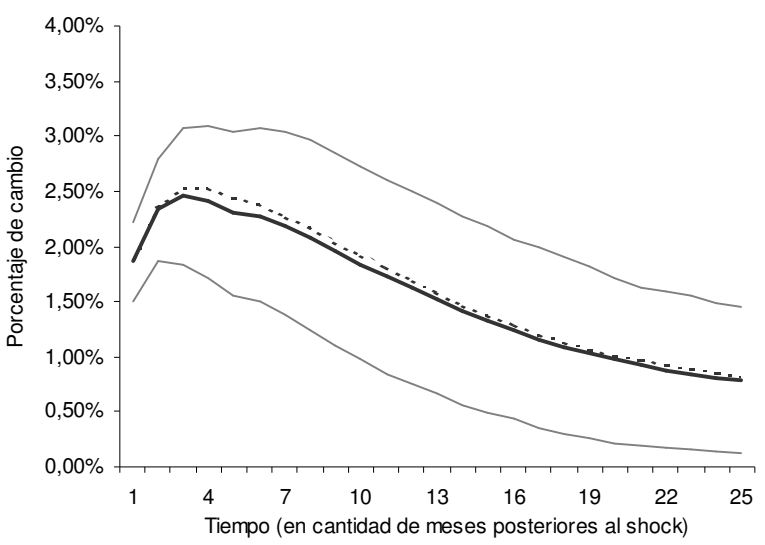

Régimen de desalineamiento bajo

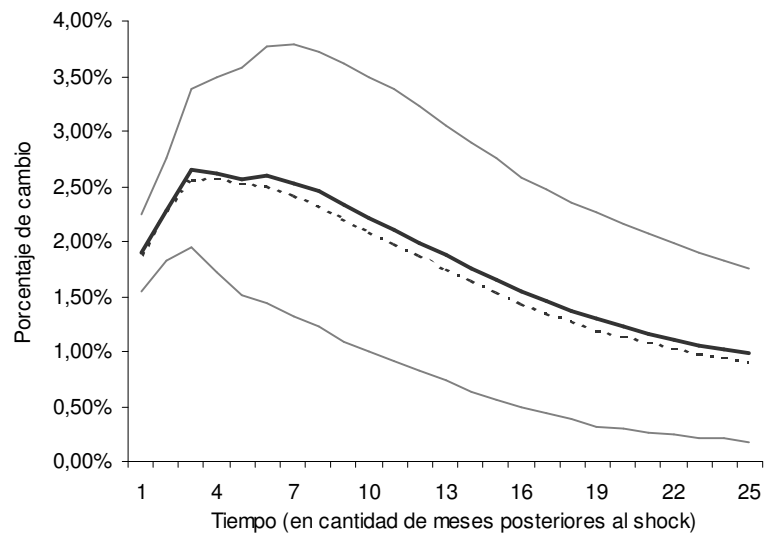

Gráfico 8. Respuesta dinámica acumulada de los precios de los commodities ante un shock en el tipo de cambio real de Estados Unidos Régimen de desalineamiento alto

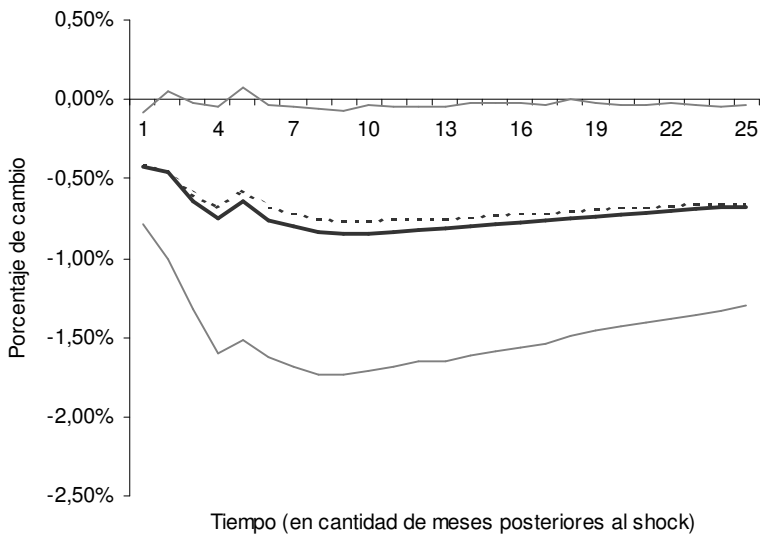

Régimen de desalineamiento bajo

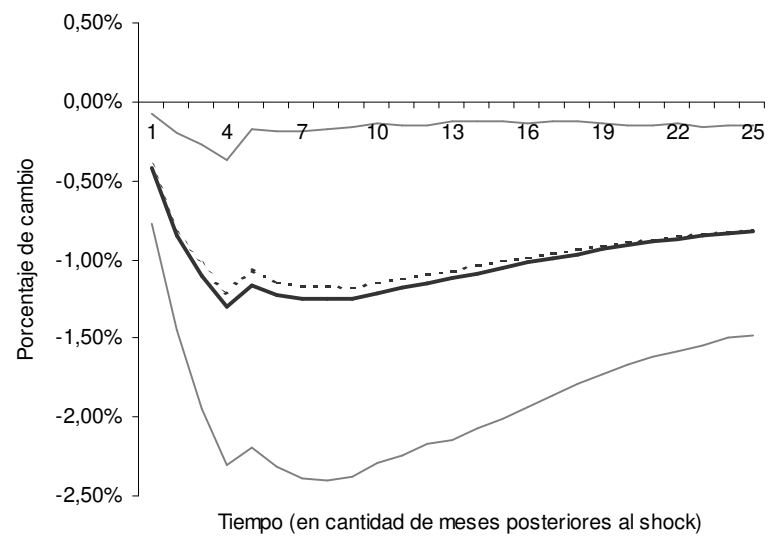


Gráfico 9. Respuesta dinámica acumulada de los precios de los commodities ante un shock en la producción industrial

Régimen de desalineamiento alto

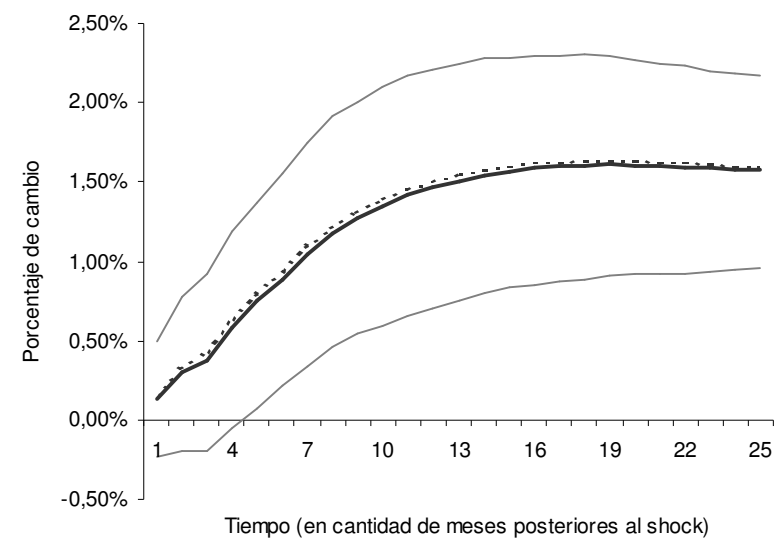

Régimen de desalineamiento bajo

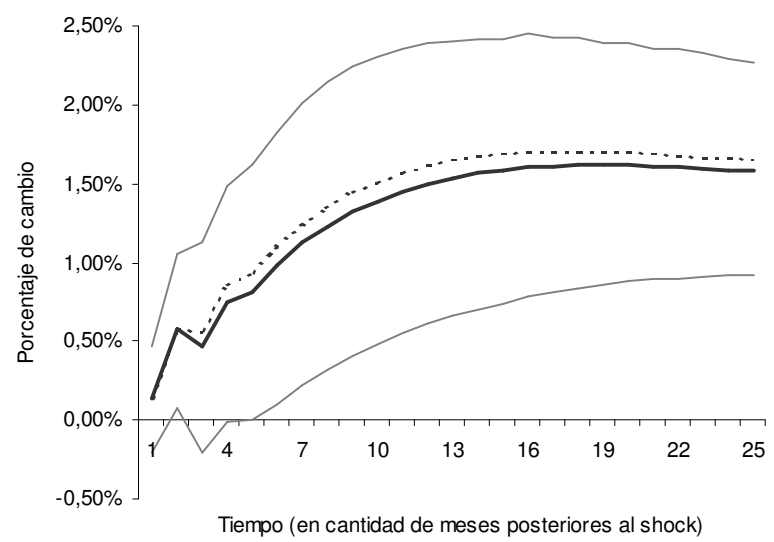

Gráfico 10. Respuesta dinámica acumulada de los precios de los commodities ante un shock en la liquidez internacional

Régimen de desalineamiento alto

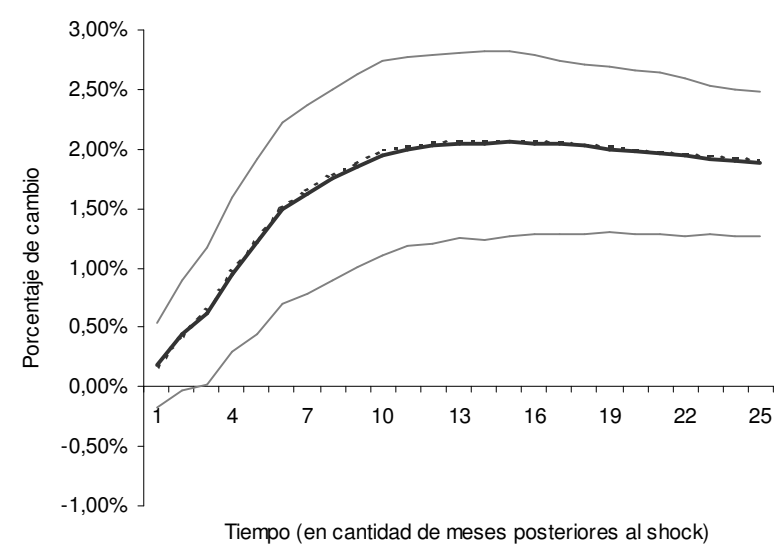

Régimen de desalineamiento bajo

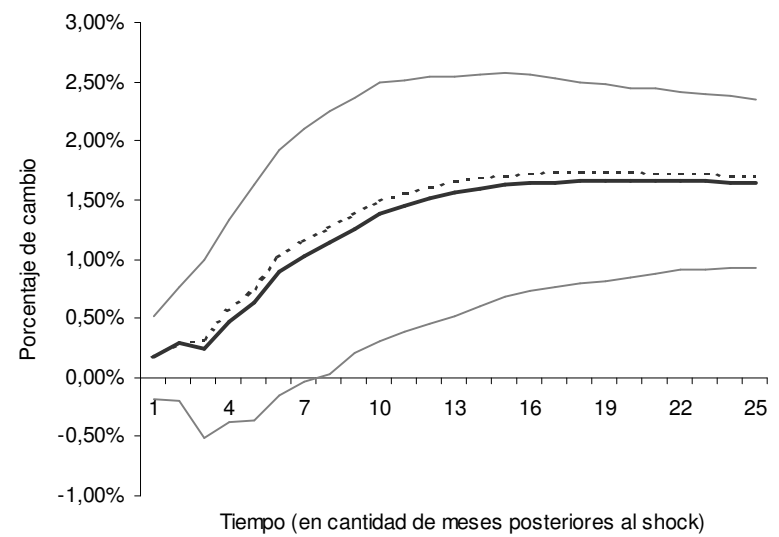

Gráfico 11. Respuesta dinámica acumulada de los precios de los commodities ante un shock en el Dow Jones

Régimen de desalineamiento alto

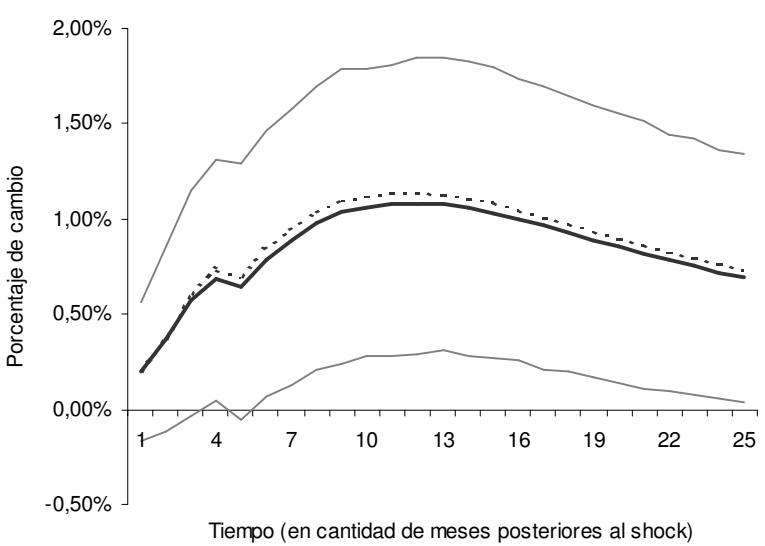

Régimen de desalineamiento bajo

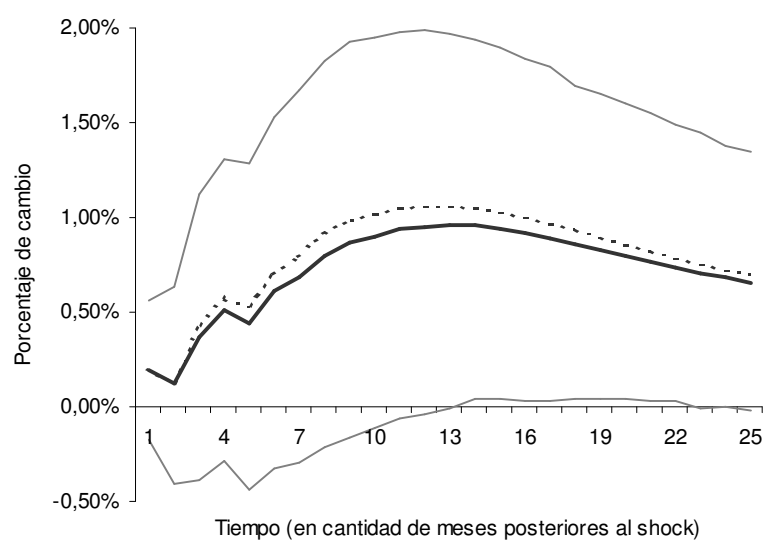


A grandes rasgos surgen dos grupos de conclusiones del análisis de los Gráficos 7 a 11: i) los signos obtenidos se corresponden con lo sugerido por la teoría y las relaciones de largo plazo; y ii) no hay grandes diferencias cualitativas en los efectos de las perturbaciones bajo los distintos regímenes.

La primera conclusión tiene que ver con los signos y formas dinámicas obtenidas en las simulaciones. En este sentido, se observa que, con excepción de lo que ocurre con el Dow Jones, los shocks en las variables del modelo influyen a los precios en la misma dirección que la señalada por las relaciones de largo plazo.

En cuanto a las magnitudes involucradas, los shocks positivos en la producción industrial y la liquidez internacional golpean en la misma dirección a los precios, con un efecto máximo en torno al $1,5 \%$ y $2 \%$. Los efectos del TCR y el Dow Jones tienen una menor importancia relativa, alcanzando un tope aproximado de $1 \%$ en el cambio en el nivel de los precios de los commodities (negativo en el primer caso y positivo en el segundo). No se observan, por otra parte, grandes asimetrías por tamaño del choque. Una perturbación de un desvío estándar en el propio precio de los commodities (Gráfico 7) se transmite con un efecto máximo de entre 2,5\% y $3 \%$ hasta los cuatro meses posteriores a su ocurrencia. Luego, decae lentamente hasta estabilizarse en torno al $1 \%$ de cambio en el nivel de los precios.

La segunda dimensión de resultados involucra la comparación de choques entre cada tipo de régimen. Al respecto, no se verificaron diferencias sustanciales en los valores de las respuestas dinámicas. Igualmente, en todos los casos, las mayores discrepancias se producen en los primeros meses posteriores al choque. La razón obedece a la actualización que se produce en el desalineamiento. Ella causa que los choques que comienzan en valores bajos de $w_{t}$ vayan modificando el misalignment y, con esto, el propio valor de la función de transición en el futuro, acercándolos con el paso del tiempo a valores más bien altos ya que esta es la tendencia más probable en el modelo no lineal.

La evaluación global de los resultados del sistema (22) a (26) parece ser muy satisfactoria en términos de correspondencia con los desarrollos teóricos, con los resultados de otros trabajos empíricos y con la motivación general dada en este estudio. Sin embargo, como se señaló anteriormente, esta línea de trabajo parece más un punto de partida que de llegada.

\section{Reflexiones finales}

Distinguir entre movimientos transitorios y permanentes de una variable es una de las tareas más arduas que enfrentan los agentes en general y los policymakers en particular. Cuando demasiados actores extrapolan linealmente hacia el futuro la evolución pasada de algun indicador, podemos estar a las puertas de una inconsistencia global de planes o falla de coordinación. No hace falta ir muy lejos en el tiempo para encontrar un nítido ejemplo: el descalabro de la crisis de las hipotecas sub-prime y el mercado crediticio norteamericano sostenido, ente otros aspectos, en la falsa creencia de que los precios de las propiedades siempre irían en ascenso.

Con este antecedente inmediato y luego de varios años de rápida suba en los precios de los commodities junto a un marcado crecimiento en monto y variedad de instrumentos de inversión de ellos derivados, es momento de preguntarse rigurosamente cuánto de este aumento se corresponde con los fundamentales y qué tanto obedece a la simple especulación financiera. Si la respuesta se trabaja adecuadamente, no necesariamente ubicará a quien intenta abordarla 
en la situación de quien anuncia el final de un ciclo alcista y aguarda, como espectador, si ocurre tal pronóstico.

Así, la motivación del estudio fue brindar un panorama balanceado reconociendo que, seguramente, hay factores estructurales que justifican en algún grado las cotizaciones recientemente observadas sin por ello ignorar que pueden existir movimientos desproporcionados en relación a los fundamentales.

También hay que reconocer que es lento el aprendizaje sobre los efectos que tienen los cambios estructurales en las variables económicas, auque se cuente incluso con metodologías econométricas capaces de aproximarlo. No se duda de que la creciente demanda actual y futura de China por más soja, cobre o petróleo impacta (e impactará) positivamente en los precios; se discute si esta demanda condice con un valor de la oleaginosa en 400 US\$ o en 600 US\$ la tonelada, con un barril a 70 US\$ o a 110 US\$. Sea cual fuese el "equilibrio" relevante, el esquema teórico y empírico adoptado tiene, como atractivo, la capacidad para explicar por qué ambos valores podrían observarse en cortos intervalos de tiempo.

Por estas razones se buscó incorporar cuestiones estructurales como la mayor importancia de los países emergentes en la economía mundial, el debilitamiento de la moneda que es patrón de las finanzas internacionales o la expansión de la liquidez internacional. En todos los casos se encontraron relaciones de corto y largo plazo acordes con la teoría económica y compatibles con una dinámica no lineal de ajuste al equilibrio. Debe subrayarse que aunque algunas de estas variables determinantes cambian lentamente, otras pueden sufrir vaivenes repentinos. Por sus efectos sobre las expectativas, estos movimientos en los fundamentales pueden actuar como catalizadores, amplificando más que proporcionalmente los defasajes iniciales.

Si hay que señalar los principales factores detrás de las alzas actuales en los precios de los commodities, son insoslayables los efectos de la abundante liquidez global, el crecimiento económico motorizado por china y la gran caída en el valor del dólar. Aun suponiendo que estos factores no se alteren en el corto plazo, la brecha entre precios y sus fundamentales no hace improbable algún tipo de corrección a futuro al menos si la referencia es la historia reciente.

Varias líneas de trabajo quedan abiertas como guía para futuras investigaciones o extensiones. Seguramente, la más relevante es la apertura del índice de commodities a una menor desagregación para permitir reacciones específicas por grupos de bienes más homogéneos 0 incluso para commodities puntuales. Esto permitiría aumentar la potencia de la metodología y acercar recomendaciones de política económica más específicas si, por ejemplo, se aproxima cabalmente la canasta de commodities relevantes de un país o región particular. Otras mejoras pueden pasar por una especificación más refinada de la relación de largo plazo, cuestión que demanda un esfuerzo para encontrar proxies factibles y relevantes.

Teniendo en mente la relación de los commodities con el crecimiento, la apreciación cambiaria - la generación de divisas e ingresos fiscales en economías emergentes, la principal recomendación de política económica que puede sugerirse de los resultados es la prudencia.

Así, mientras se trabaja en aquellas medidas capaces de incrementar la diversificación productiva de las economías dependientes de los commodities, los policymakers deberían pensar a cada suba en los precios como transitoria y tomar a cada shock adverso como permanente. 
Apartarse de este principio de estricta prudencia requeriría mecanismos de identificación de los shocks suficientemente testeados y confiables. La vocación de este estudio ha sido aportar elementos para un debate sobre tales mecanismos.

Mientras este avance en el conocimiento se materializa, la actual fase alta del ciclo de precios requiere una adecuada gestión de los riesgos macroeconómicos en los países productores con menor desarrollo relativo, que debería apuntar a minimizar los costos de las reversiones futuras. 


\section{Referencias}

Bastourre, D., J. Carrera y J. Ibarlucia (2008) "Commodity Prices in Argentina, What Does Move the Wind?", Money Affairs, CEMLA (en proceso de publicación).

Beenstock, M. (1988) "An Econometric Investigation of North-South Interdependence", en D. Currie y D. Vines (eds.) Macroeconomic Interactions between North and South, Cambridge University Press, Cambridge, U.K.

Bienkowski, N. (2007) "Exchange Trade Commodities", The London Bullion Association.

Black, F. (1976) "The Pricing of Commodity Contracts", Journal of Financial Economics, Vol. 3, 167-179.

Bleaney M. y D. Greenaway (1993) "Long-Run Trends in the Relative Price of Primary Commodities and in the Terms of Trade of Developing Countries", Oxford Economic Papers, New Series, Vol. 45, 349-363.

Borensztein E. y C. M. Reinhart (1994) "The Macroeconomic Determinants of Commodity Prices", IMF Staff Papers, Vol. 41, 236-258.

Böwer, U., A. Geis y A. Winkler (2007) "Commodity Price Fluctuations and Their Impact on Monetary and Fiscal Policies in Western and Central Africa", Occasional Paper Series No. 60, European Central Bank, Abril.

Bray, M. y N. Savin (1986) "Rational Expectations Equilibria, Learning, and Model Specification", Econometrica, Vol. 54, 1129-1160.

Camacho, M (2004) "Vector Smooth Transition Regression Models for US GDP and the Composite Index of Leading Indicators", Journal of Forecasting, Vol. 23, 173-196.

Cashin, P. y C. J. McDermott (2002) "The Long-Run Behavior of Commodity Prices: Small Trends and Big Variability", IMF Staff Papers, Vol. 49, 175-199.

Chan, K. y H. Tong (1986) "On Estimating Thresholds in Autoregressive Models", Journal of Time Series Analysis", Vol. 7, 179-191.

Chen, S., y J. Wu (2000) "A Re-Examination of Purchasing Power Parity in Japan and Taiwan" Journal of Macroeconomics, Vol. 22, 271-284.

Cheung, C. y S. Morin (2007) "The Impact of Emerging Asia on Commodity Prices", Trabajo Presentado en la XII Reunión de la Red de Investigadores de Banca Central, Banco de España, Noviembre de 2007.

Cuddington, J. y C. Urzúa (1989) "Trends and Cycles in the Net Barter Terms of Trade: a New Approach", Economic Journal, Vol. 99, 426-442.

De Gregorio, J., H. González y F. Jaque (2005) "Fluctuaciones del Dólar, Precio del Cobre y Términos del Intercambio", Documento de Trabajo del Banco Central de Chile No. 310, Febrero de 2005.

De Gregorio, J., O. Landerretche y C. Neilson (2007) "Another Pass-Through Bites the Dust? Oil Prices and Inflation", Documentos de Trabajo del Banco Central de Chile, No. 417, Mayo de 2007. 
Deaton, A. (1999) "Commodity Prices and Growth in Africa", Journal of Economic Perspectives, Vol. 13, 23-40.

Deaton, A. y G. Larroque (1992) "On the Behaviour of Commodity Prices", The Review of Economic Studies, Vol. 49, 175-199.

DeLong, J., A. Shleifer, L. Summers y R. Waldmann (1990a) "Noise Trader Risk in Financial Markets", Journal of Political Economy, Vol. 98, 703-738.

DeLong, J., A. Shleifer, L. Summers y R. Waldmann (1990b) "Positive Feedback Investment Strategies and Destabilizing Rational Speculation”, Journal of Finance, Vol. 45, 379-395.

Deutsche Bank (2005) "An Investor Guide to Commodities”, M. Lewis (ed.), Abril de 2005.

Domanski, D. y A. Healt (2007) "Financial Investors and Commodity Markets", BIS Quarterly Review, Marzo de 2007, 53-67.

Dooley, M. y P. Garber (2005) "Is It 1958 or 1968 ? Three Notes on the Longevity of the Revived Bretton Woods System", Brookings Papers on Economic Activity, Vol. 2005, 147-209.

Dornbusch, R. (1985) "Policy and Performance Links Between LDC Debtors and Industrial Nations", Brookings Papers on Economic Activity, Vol. 1985, 303-368.

Engel, E. (1895): "Die Productions- und Consumptionsverhaltnisse Des Konigreichs Sachsen", International Statistical Bulletin Institute, Vol. 9, 1-74, version original de 1857.

Erb, C. y C. Harvey (2005) "The Tactic and Strategic Value of Commodity Futures", NBER Working Paper No. 11222.

FMI (2006) "The Boom in Nonfuel Commodity Prices: Can It Last?", World Economic Outlook (Capítulo 5) , Septiembre de 2006.

FMI (2007) "Global Prospects and Policies", World Economic Outlook, (Capítulo 1), Octubre de 2007.

Fourgeaud, C., C. Gourieroux y J. Pradel (1986) "Learning Procedures and Convergence to Rationality", Econometrica, Vol. 54, 845-868.

Frankel, J. (2006) "The Effect of Monetary Policy on Real Commodity Prices", NBER Working Paper No. 12713.

Frankel, J y K. Froot (1987a) "Using Survey Data to Test Standard Propositions regarding Exchange Rate Expectations", American Economic Review, Vol. 77, 133-153.

Frankel, J. y K. Froot (1987b) "Short-Term and Long-Term Expectations of the Yen/Dollar Exchange Rate: Evidence from Survey Data”, NBER Working Paper No. 2216.

Frankel. J y K. Froot (1990) "The Rationality of the Forex Exchange Rate, Chartists, Fundamentalist and Trading in The Forex Exchange Rate Market, American Economic Review, Vol. 80, 181-185.

Franses, P. y D. van Dijk (2000) Nonlinear Time Series Models in Empirical Finance, Cambridge University Press, Cambridge.

Gilbert, C. (1989) "The Impact of Exchange Rates and Developing Country Debt on Commodity Prices”, The Economic Journal, Vol. 99, 773-784. 
Gorton, G. y K. Rouwenhorst (2004) "Facts and Fantasies about Commodity Futures", NBER Working Paper No. 10595.

Granger, C. y T. Teräsvirta (1993) Modeling Nonlinear Economic Relationships, Oxford University Press, New York.

Grilli, E. y M. Yang (1988) "Primary Commodity Prices, Manufactured Goods, Prices and Terms of Trade of Developing Countries: What the Long Run Shows", World Bank Economic Review, Vol. 2, 1-48.

Hamilton, B. (2001) "Using Engel's Law to Estimate CPI Bias", American Economic Review, Vol. 91, 619-630.

Harberger, A. (1950) "Currency Depreciation, Income and the Balance of Trade", Journal of Political Economy, Vol. 58, 47-60.

Holmes, D. (2006) “A Financial Feast: a-la-carte Commodity Investing”, Alchemy, Issue 43, The London Bullion Market Association.

Houthakker, H. (1987) "Engel's Law”, en J. Eatwell, M. Milgate y P. Newman (eds.) The New Palgrave: A Dictionary of Economics", London, Macmillan, 1987, 143-144.

HSBC (2007) "When All the Boats Floated (a True Story)", Emerging Markets Primal Research. Macro, Febrero de 2007.

Ito, K. (1990) "Foreign Exchange Rate Expectations", American Economic Review, Vol. 80, 434449.

Johnson, S. (2007) "Food and Biofuels: The Price of Success", IMF Survey Magazine, Diciembre de 2007.

Kaplinsky, R. (2006) "Revisiting the Revisited Terms of Trade: Will China Make a Difference?", World Development, Vol. 34, 981-995.

Keynes, J. M. (1930) A Treatise on Money, MacMillan, London.

Keynes, J, M. (1936) The General Theory of Unemployment, Interest and Money, Harcourt, Brace and World, New York.

Kolb, R. (1997) Understanding Future Markets, Blackwell Publishers, Oxford.

Koop, G., H Pesaran, y S. Potter (1996) "Impulse Response Analysis in Nonlinear Multivariate Models", Journal of Econometrics, Vol. 74, 119-47.

Korhonen, I. (2004) "Does Democracy Cure a Resource Curse?" BOFIT Discussion Paper $18 / 2004$.

Laursen, S. y L. Metzler (1950) "Flexible Exchange Rates and the Theory of Employment", Review of Economic and Statistics, Vol. 32, 281-299.

Lewis, M. (2005) "The History and Development of Commodities Exchanges" en M. Lewis (ed.) An Investor Guide to Commodities, Deutsche Bank, Abril de 2005.

Luukkonen, R., P. Saikkonen y T. Teräsvirta (1998) "Testing Linearity Against Smooth Transition Autoregressive Models", Biometrica, Vol. 75, 491-499. 
Lutz, M. (1999) "A General Test of the Prebisch-Singer Hypothesis", Review of Development Economics, Vol. 3, 44-57.

McNee, A. (2006) "Investors Slake Commodities Thirst with Structured Products", The Banker, Julio de 2006.

Mendoza, O. (2004) "Las Asimetrías del Pass-Through en Venezuela", Colección Economía y Finanzas: Serie Documentos de Trabajo No. 62, Banco Central de Venezuela.

Michael, P., A. Nobay y D. Peel (1997) "Transactions Costs and Nonlinear Adjustments in Real Exchange Rates: An Empirical Investigation", Journal of Political Economy, Vol. 105, 862-879.

Monoyios, M. y L. Sarno (2002) "Mean Reversion in Stock Index Futures: A Nonlinear Analysis", The Journal of Futures Markets, Vol. 22, 285-314.

Morgan Stanley (2007) "How Big Could Sovereign Wealth Funds Be by 2015?", Morgan Stanley Research Global, Mayo de 2007.

Ocampo, J. y M. Parra (2003) "Returning to a Eternal Debate: The Terms of Trade in the Twentieth Century", Serie Estudios e Informes Especiales No. 5, CEPAL, Santiago.

Powell, A. (1991) "Commodity and Developing Countries Terms of Trade: What Does the Long Run Shows?", Economic Journal, Vol. 101, 1485-1496.

Prebisch, R. (1950) "The Economic Development of Latin America and Its Principal Problems", New York, United Nations; Reimpreso al Español en Desarrollo Económico, Vol. 26, 251302.

Ridler, D. y C. Yandle (1972) "A Simplified Method of Analyzing the Effects of Exchange Rates on Exports of a Primary Commodity", IMF Staff Papers, Vol.19, 559-578.

Sachs, J. (1981) "The Current Account and Macroeconomic Adjustment in the 1970's", Brooking Papers on Economic Activity, Vol. 1981, 201-68.

Sachs, J. y A. Warner (1995) "Natural Resource Abundance and Economic Growth", NBER Working Paper No. 5398.

Sala-i-Martin, X. y A. Subramanian (2003) "Addressing the Natural Resource Curse: An Illustration from Nigeria", IMF Working Paper 03/139, Julio.

Sarno, L., G. Valente y H. Leon (2006) "Nonlinearity in Deviations from Uncovered Interest Parity: An Explanation of the Forward Bias Puzzle", Review of Finance, Vol. 10, 443-482.

Shleifer, A. y L. Summers (1990) "The Noise Trader Approach to Finance", Journal of Economic Perspectives, Vol. 2, 19-33.

Simon, H. (1957) Models of Man, Wiley, New York, NY.

Singer, H. (1950) "The Distribution of Gains between Investing and Borrowing Countries", American Economic Review, Vol. 40, 473-485.

Stevens, P. (2003) "Resource Impact Curse or Blessing? A Literature Survey", Journal of Energy Literature, Vol. 9, 3-42.

Stock, J. y M. Watson (1993) "A Simple Estimator of Cointegrating Vectors in Higher-Order Integrated Systems”, Econometrica, Vol. 6, 738-820. 
Taylor, M.A. y H. Allen (1992) "The Use of Technical Analysis in the Foreign Exchange Rate Market", Journal of International Money and Finance, Vol. 11, 304-314.

Taylor, M.P., D. Peel y L. Sarno (2001) "Nonlinear Mean-Reversion in Real Exchange Rates: Towards a Solution to the Purchasing Power Parity Puzzles", International Economic Review, Vol. 42, 1015-1042.

Teräsvirta, T. (1994) "Specification, Estimation and Evaluation of Smooth Transition Autoregressive Models", Journal of the American Statistical Association, Vol. 89, 208218.

Tong, H. (1978) On a Threshold Model in Pattern Recognition and Signal Processing, C.H. Chen (ed), Sijhoff and Noordhoff, Amsterdam.

Tong, H. (1990) Nonlinear Time Series: a Dynamical System Approach, Claredon Press, Oxford.

Tornell, A. y P. Lane (1999) "The Voracity Effect", American Economic Review, Vol. 89, 22-46.

Tsay, R. (1989) "Testing and Modeling Threshold Autoregressive Processes", Journal of the American Statistical Association, Vol. 84, 231-240.

UNCTAD (2006) "The Emerging Biofuels Market: Regulatory, Trade and Development Implications", United Nations.

van Dijk, D. (2001) "Smooth Transition Models: Extensions and Outlier Robust Inference", Tinberg Institute: Amsterdam.

van Dijk, D., T. Teräsvirta, y P. Franses (2002) "Smooth Transition Autoregressive Models - A Survey of Recent Developments", Econometric Reviews, Vol. 21, 1-47.

Weise, C. (1999) "The Asymmetric Effects of Monetary Policy: a Nonlinear Vector Autoregression Approach", Journal of Money, Credit and Banking, Vol. 31, 85-108.

Westerhoff, F. y S. Reitz (2005) "Commodity Price Dynamics and the Nonlinear Market Impact of Technical Traders: Empirical Evidence for the US Corn Market”, Physica A 349, Vol. 2005, 641-648. 